\title{
Role of non-coding RNAs in tumor progression and metastasis in pancreatic cancer
}

\author{
Lorenzo F. Sempere ${ }^{1}$ (D) Katherine Powell ${ }^{1} \cdot$ Jatin Rana ${ }^{2} \cdot$ Andrew A. Brock $^{3} \cdot$ Thomas D. Schmittgen $^{3}$
}

Received: 4 August 2021 / Accepted: 13 September 2021 / Published online: 30 September 2021

(c) The Author(s) 2021

\begin{abstract}
Pancreatic ductal adenocarcinoma (PDAC) is one of the most lethal types of cancer with an overall 5-year survival rate of less than $10 \%$. The 1-year survival rate of patients with locally advanced or metastatic disease is abysmal. The aggressive nature of cancer cells, hypovascularization, extensive desmoplastic stroma, and immunosuppressive tumor microenvironment (TME) endows PDAC tumors with multiple mechanisms of drug resistance. With no obvious genetic mutation(s) driving tumor progression or metastatic transition, the challenges for understanding the biological mechanism(s) of these processes are paramount. A better understanding of the molecular and cellular mechanisms of these processes could lead to new diagnostic tools for patient management and new targets for therapeutic intervention. microRNAs (miRNAs) are an evolutionarily conserved gene class of short non-coding regulatory RNAs. miRNAs are an extensive regulatory layer that controls gene expression at the posttranscriptional level. This review focuses on preclinical models that functionally dissect miRNA activity in tumor progression or metastatic processes in PDAC. Collectively, these studies suggest an influence of miRNAs and RNA-RNA networks in the processes of epithelial to mesenchymal cell transition and cancer cell stemness. At a cell-type level, some miRNAs mainly influence cancer cell-intrinsic processes and pathways, whereas other miRNAs predominantly act in distinct cellular compartments of the TME to regulate fibroblast and immune cell functions and/or influence other cell types' function via cell-to-cell communications by transfer of extracellular vesicles. At a molecular level, the influence of miRNA-mediated regulation often converges in core signaling pathways, including TGF- $\beta$, JAK/STAT, $\mathrm{PI} 3 \mathrm{~K} / \mathrm{AKT}$, and NF-KB.
\end{abstract}

Keywords microRNA (miR · miRNA) · Long non-coding RNAs (lncRNA) · Circular non-coding RNA (circRNA) · Extracellular vesicles

\section{Introduction}

Pancreatic ductal adenocarcinoma is one of the most lethal types of cancer with an overall 5-year survival rate of less than $10 \%$ [1]. Virtually all long-term survivors are patients with early stage disease for whom surgical resection and adjuvant systemic therapy provides a potential cure [2,3]. Due to late clinical presentation, about $30 \%$ of patients

Lorenzo F. Sempere

semperel@msu.edu

1 Precision Health Program, College of Human Medicine, Michigan State University, East Lansing, MI 48824, USA

2 Department of Medicine, College of Human Medicine, Michigan State University, East Lansing, MI 48824, USA

3 Department of Pharmaceutics, College of Pharmacy, University of Florida, Gainesville, FL 32610, USA are diagnosed with locally advanced disease and about $50 \%$ of patients with metastatic disease [2]. The 1-year survival rate of these advanced stage cases is abysmal [2, $3]$. Modifications in systemic chemotherapy regimens such as FOLFIRINOX (5-fluorouracil, folinic acid, irinotecan, and oxaliplatin) or gemcitabine-containing combination treatments have resulted in a modest improvement of patient outcome in recent years [2]. Targeted therapies and immunotherapies, which have improved outcome in other cancer types, have failed to provide a clear clinical signal in PDAC $[2,4]$. The aggressive nature of cancer cells, hypovascularization, extensive desmoplastic stroma, and immunosuppressive tumor microenvironment (TME) endows PDAC tumors with multiple mechanisms of drug resistance $[3,5,6]$. Combination strategies that target cancer cells and elements of the TME may offer new opportunities and hope in PDAC. Myeloid cell-modifying agonist 
CD40 antibody, stroma-modifying angiotensin-receptor blocker Losartan, CXCR4 antagonist in combination with PD-1 blockade, and connective tissue growth factor inhibitors are showing promising results in on-going clinical trials $[2,7,8]$. In addition, recent studies continue to investigate the clinical value of circulating tumor DNA and other molecular biomarkers for early disease detection $[9,10]$.

PDAC has a limited number of driver genes, with frequent and predominant mutations in KRAS (>90\%), CDKN2A ( 90\%), TP53 ( 70\%), and SMAD4 ( 55\%) $[3,11]$. With no obvious genetic mutation(s) driving tumor progression or metastatic transition, the challenges for understanding the biological mechanism(s) of these processes are paramount. These require a comprehensive and integrative analysis of dynamic changes in gene dosage, transcriptome, epigenome, core signaling, and metabolomic pathways that collectively contribute to tumor evolution and metastatic spread [11-15]. A better understanding of the molecular and cellular mechanisms of these processes could lead to new diagnostic tools for patient management and new targets for therapeutic intervention. microRNAs (miRNAs) are an evolutionarily conserved gene class of short non-coding regulatory RNAs [16-18]. miRNAs are an extensive regulatory layer that controls gene expression at the posttranscriptional level. miRNA expression and function has been linked to different aspects of PDAC biology and disease progression [18-23]. This review focuses on preclinical models that functionally dissect miRNA activity in tumor progression or metastatic processes. Collectively, these studies suggest an influence of miRNAs and RNA-RNA networks in the processes of epithelial to mesenchymal cell transition (EMT), proliferation, and cancer cell stemness. At a celltype level, some miRNAs mainly influence the processes and pathways in a cancer cell-intrinsic manner, whereas other miRNAs predominantly act in distinct cellular compartments of the TME to regulate fibroblast and immune cell functions and/or to dictate other cell types' functions via cell-to-cell communication by transfer of extracellular vesicles (EVs). At a molecular level, the influence of miRNA-mediated regulation often converges in core signaling pathways, including TGF- $\beta$, JAK/STAT, PI3K/ $\mathrm{AKT}$, and NF-кB. In the following sections, we discuss salient examples of these mechanisms of action and crosstalk between cancer cells and other cell types in the TME. We focus our discussion on recent studies in which there is an in vivo demonstration of miRNA activity in tumor progression and/or metastatic processes. This in vivo demonstration may involve the use of genetically engineered mouse models, organoid or cell line xenograft models, and/or patient-derived tumor models.

\section{2 microRNA biogenesis and dysregulation during PDAC progression}

The mature and biological active miRNA consists of a 19-24 nucleotide-long RNA molecule resulting from a stepwise processing of a much longer mRNA-like primary transcript (Fig. 1). The immense majority of the mRNA-like primary transcripts are transcribed by RNA polymerase II [16, 17]. Some miRNAs are arranged in tightly linked gene clusters, and this primary transcript (pri-miRNA) may contain the precursor hairpin RNA (pre-miRNA) for several miRNAs [24]. Transcription of either a single miRNA or a miRNA gene cluster may initiate from a proximal promoter to the miRNA gene or distal promoter driving expression of the host gene $[16,17]$. In the nucleus, RNAse III DROSHAcontaining RNA microprocessor complex cleaves precursor hairpin RNA that is exported to the cytoplasm by XPO5facililated pathway [17]. In the cytoplasm, another RNAse III DICER cleaves the final mature RNA molecule that becomes functionally active when loaded into the ARGONAUTE-containing miRNA-induced silencing complex (miRISC). The miRNA serves as a guide to direct miRISC in close proximity to target mRNA by binding to partially complementary sites, typically on the $3^{\prime}$ UTR. The interaction of miRISC with other mRNA-bound protein complexes (e.g., CCR4-NOT, eIF4A-G) leads to mRNA decay, mRNA cleavage, and/or inhibition of translation initiation that ultimately results in a decreased protein output of the target gene(s) [16, 17]. In most cases, miRNA binding to the mRNA is driven by the seed region ( $2^{\text {nd }}$ to $8^{\text {th }}$ nucleotides of the miRNA) which allows for interactions of a single miRNA with tens or hundreds of mRNAs. The relative abundance of miRNA to target mRNA and number of binding sites and binding affinity determines the extent of protein output reduction for each target gene [18]. A single miRNA can influence cellular programs by profound downregulation of a handful of key targets and/or by more modest but coordinated downregulation of a larger number of target genes. About 2,000 bona fide miRNA genes have been identified in the human genome [24]; it is estimated that all miRNAs expressed in a particular cell can modulate the expression of up to $60 \%$ of the protein-encoding genes and correspondingly influence a large number of cellular programs $[16,17,25]$.

Dysregulation of miRNA expression and activity in PDAC can occur at different levels from chromosomal alteration (e.g., gain or loss of copy numbers), epigenetic and transcriptional regulation of the pri-miRNA, processing of pri-miRNA, export and processing of pre-miRNA, and/or physical interaction with other non-coding RNAs (Fig. 1). Dysregulation of miRNA expression has been extensively characterized between normal pancreas and PDAC tumor specimens, chronic pancreatitis and PDAC tumor specimens, 


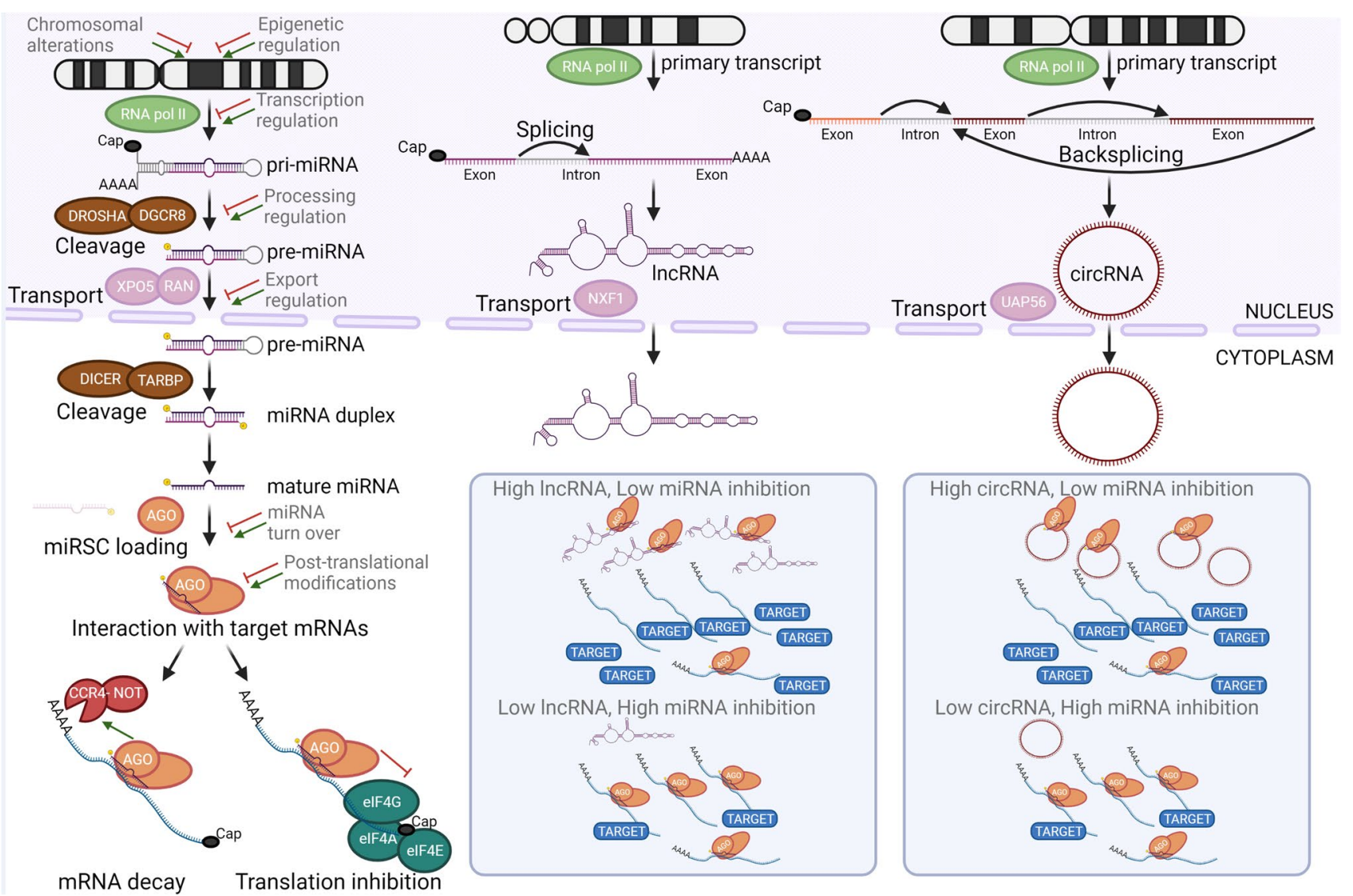

Fig. 1 Biogenesis of miRNAs and other non-coding RNAs. Key steps of biogenesis of miRNAs and miRNA-interacting lncRNAs and circRNAs. Several mechanisms are known that affect regulation of miRNA expression and/or activity. Nuclear export is key step for cytoplasmic interaction of miRNAs and these other classes of ncRNAs that sequester miRNAs away from target mRNAs and indirectly increase protein product of the target gene. Abbreviations: AGO, argonaute RISC component; circRNA, circular non-coding RNA; CCR4-NOT, carbon catabolite repression-negative on TATA-less

and PDAC precursor lesions and PDAC tumor specimens at different clinical stages. These tissue correlative studies have led to the identification of specific miRNAs or miRNA signatures that could serve as diagnostic and/or prognostic biomarkers [18, 20,21]. While there is no complete overlap among different studies, altered expression of miRNAs (e.g., let-7a, miR-10b, miR-21, miR-217) has been frequently associated with disease progression and/or metastatic disease. Guided by these clinical observations, many of these miRNAs have been subjected to a battery of in vitro and in vivo functional assays to dissect the etiological contribution of each of these miRNAs to a specific metastatic process [22, 23]. In most of these studies, in vivo evidence was obtained in different xenograft models in which immunocompromised mouse host is inoculated with human PDAC cells. In xenograft models in which the PDAC cells are inoculated subcutaneously (animal's flank) or orthotopically (pancreas), the complex; DICER, ribonuclease III Dicer1; DGRCR8, DiGeorge syndrome critical region gene 8, microprocessor complex subunit; DROSHA, ribonuclease III Dicer1; eiF4, eukaryotic translation initiation factor 4; lncRNA, long non-coding RNA; miRISC, miRNA-induced silencing complex; NXF1, nuclear RNA export factor 1; RAN, member of RAS oncogene family, small nuclear GTPase; RNA pol II, RNA polymerase II; miRNA, microRNA; TARBP, TAR (HIV-1) RNA-binding protein 1; UAP56, U2AF65-associated protein 56 also known as DDX39B (DExD-box helicase 39B); XPO5, exportin 5

effects of miRNA activity modulation can be interrogated at several stages of disease progression from growth of the primary tumor to increase migration and invasive behavior to intravasation and extravasation and colonization and growth at distant sites. In xenograft models in which the PDAC cells are inoculated intrasplenically (spleen) or intravenously (tail vein), the effects of miRNA activity modulation can be interrogated at more advance stages of disease progression from extravasation to colonization and growth at distant sites. This in vivo evidence suggests that some miRNAs (e.g., miR-200b, miR-323-3p, miR-367, miR-489) appears to exclusively or predominantly influence a specific step of the metastatic process, whereas other miRNAs (e.g., let-7a, miR-10b, miR-21, miR-29b, miR-34a) may influence multiple cellular processes (proliferation, survival, and/or drug resistance) that collectively contribute to tumor progression and metastatic spread. 


\section{3 miRNA-mediated regulation of epithelial to mesenchymal transition}

Epithelial to mesenchymal transition (EMT) is a process associated with progression and metastatic spread, by which cancer cells acquire increased motility and invasive behavior [26]. TGF- $\beta$ and HIF signaling are known inducers and regulators of EMT in PDAC [26]. Ultimately, the integration of these and other input signals leads to upregulation of ZEB1/2, TWIST1, and/or SNAI1/2: transcription factors that promote mesenchymal program and downregulation of epithelial cell adhesion protein, E-cadherin. Several miRNAs have been identified in PDAC with a major or predominant role in regulating EMT (Fig. 2; refs [27-46]). miR-202-5p, -323-3p, -663a, and -4787-5p put a break on the EMT program by inhibiting expression of key components of TGF- $\beta$ signaling pathway [28-30], whereas miR-367 promotes EMT by inhibiting expression of SMAD7, negative regulator of TGF- $\beta$ signaling [31]. Similarly, miR-301a promotes EMT by relaying hypoxic HIF2 $\alpha$ signal to increase JAK1/STAT3 signaling via inhibition of direct target SOCS5 [32] and to decrease transcription program of direct target TP63 [33]. miR-302a relays pro-metastatic signal of BRM, a catalytic ATPse subunit of the SWI/SNF chromatin remodeling complex, to increase JAK2/STAT3 signaling also via inhibition of direct target SOCS5 [39]. In contrast, miR-448 activity suppresses metastatic spread by downregulating expression of direct target gene JAK1 and dampening pro-EMT JAK/STAT3 signaling [40]. miR-10a decreases Hippo signaling and its negative regulation of EMT and cancer cell stemness via inhibition of direct target $W W C 2$ [46]. Increased activity of WWC2 leads to phosphorylation and activation of LATS1/2, which in turn prevents nuclear translocation and activity YAP/TAZ transcription complex [46]. The miR-200 family members are arranged in two gene clusters $(M I R 200 b \sim M I R 200 a \sim M I R 429$ at chromosome 1 and MIR200c MIR141 at chromosome 12) in humans. miR-200 family members are considered safeguards of epithelial cell program and identity by inhibiting expression of direct target genes ZEB1 and ZEB2 [34-36]. Unlike breast and other cancer types, individual activity of these miRNAs leads to different regulation of EMT and tumor suppressive properties in orthotopic Panc-1 xenograft tumor model [34]. Enforced expression of miR-141 or miR-429 results in a significant inhibition of tumor growth in this orthotopic model, whereas that of other miR-200 family members or their combination with miR-141 and miR-429 does not [34]. miR-141 tumor suppressive and anti-metastatic function is also mediated by direct inhibition of MAP4K4 [37] and WIPF1 [38], respectively. There is a differential association between expression changes of individual miR-200 family members and that of ZEB1, ZEB2, and E-cadherin in PDAC clinical samples and patient-derived xenograft tumors [34]. In a

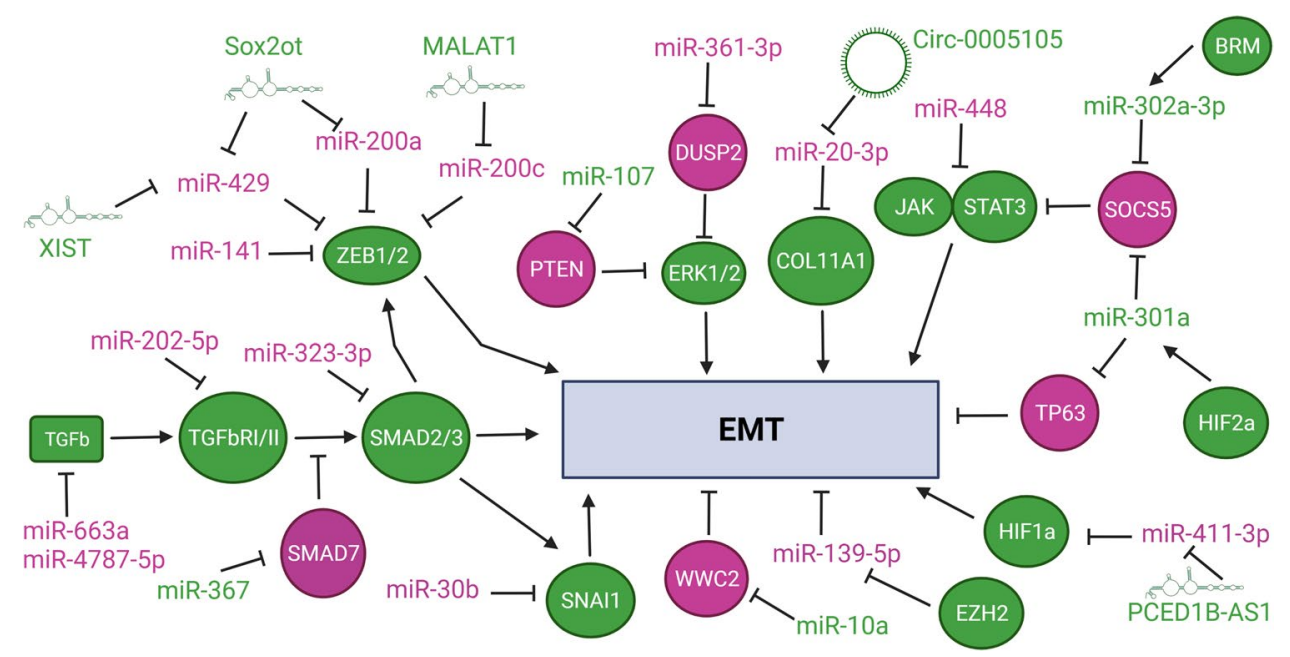

Fig. 2 miRNA-mediated regulatory networks influencing EMT program. miRNAs and protein-encoding genes (ovals) depicted in violet maintain or favor epithelial program (anti-EMT, tumor suppressive), whereas miRNAs, IncRNAs, circRNAs, and protein-encoding genes in green favor mesenchymal program (pro-EMT, tumor promoting). In other contexts, these miRNAs may influence other cellular programs and decisions. Abbreviations: BRM, Brahma; COL11A1 collagen 11A1 ; DUSP2, dual specificity phosphatase 2, ERK1/2, extracellular signal-regulated kinases; EZH2, enhancer of zeste 2 polycomb repressive complex 2 subunit; HIF1a/2a, hypoxia inducible factor $1 / 2$ subunit alpha; JAK, Janus kinase; PTEN, phosphatase and tensin homolog; SMAD2/3/7, similar to gene product of $C$. elegans Sma (Small) and Drosophila Mad (mothers against decapentaplegic) 2/3/7; SNAI1, snail family transcriptional repressor 1; SOCS5, suppressor of cytokine signaling 5; STAT3, signal transducer and activator of transcription 3; TGFb, tumor growth factor $\beta$; TGFbRI/ II, TGF- $\beta$ receptor I/II; TP63, tumor protein 63 ; ZEB1/2, zinc finger E-box binding homeobox $1 / 2$ 
cohort of 31 PDAC primary tumors, there is no consistent trend of downregulation for all miR-200 family members, but there is a positive correlation between expression of all miR-200 family and that of E-cadherin [34]. Negative association is only statistically significant for expression of miR-200a and miR-141 in relation to that of ZEBI and $Z E B 2$ [34]. These results add a level of complexity to the regulation of EMT and perhaps other metastatic processes by each of these related miRNAs and suggest the importance of context, timing, and differential inhibition of key target genes (other than ZEB1/ZEB2).

\section{4 miRNA-mediated regulation of extracellular matrix remodeling, invasion, and metastatic spread}

The extracellular matrix (ECM) is composed proteoglycans, proteins, and matricellular associated proteins. ECM remodeling promotes growth, survival, and invasion of PDAC cells at the primary tumor and facilitates colonization at premetastatic niches in the liver and other distant organs [47]. Some miRNAs have a direct role in limiting the tumorigenic remodeling of the ECM (Fig. 3, refs [48, 49]) by modulating the expression of matrix metalloproteinases (MMPs) and disintegrin and metalloproteinases (ADAMs). miR-29c activity inhibits the expression of direct target gene $M M P 2$ [48]. Sequential selection of Hs766t cells with preferential metastasis to the liver shows the requirement of miR-29c to limit metastatic spread [48]. It is not clear if miR-29c is more crucial at escaping the primary tumor site and/or reducing colonization and growth at distant metastatic site. miR-489 activity inhibits the expression of direct target genes $M M P 7$ and ADAM9 and is required for limiting colonization and growth at distant metastatic sites [49]. Other miRNAs influence cellular processes of growth, invasion, and/or metastatic spread that indirectly affect cancer cellmediated remodeling of the ECM, interaction with ECM elements, and/or mechanosensing of stroma stiffness (Fig. 3, refs [27, 50-65]). miR-664 and miR-942 modulate cancer cell growth and invasion by direct inhibition of common target gene transcription factor PAX6 [53, 54]. Similarly, miR-132, miR-212-3p, and miR-494 modulate invasion and metastatic spread by direct inhibition of common target gene transcription factor FOXM1 [55, 56]. SMAD4 positively regulates expression of miR-494 via canonical TGF- $\beta$ signaling pathway [56]. Genetic loss of SMAD4 downregulates miR494 expression and leads to upregulation of FOXM1 along with potentiation of WNT signaling by FOXM1-mediated nuclear translocation of $\beta$-catenin [56]. This illustrates the complexity and duality of TFG- $\beta$ signaling in suppressing tumor growth in early stages of carcinogenesis while

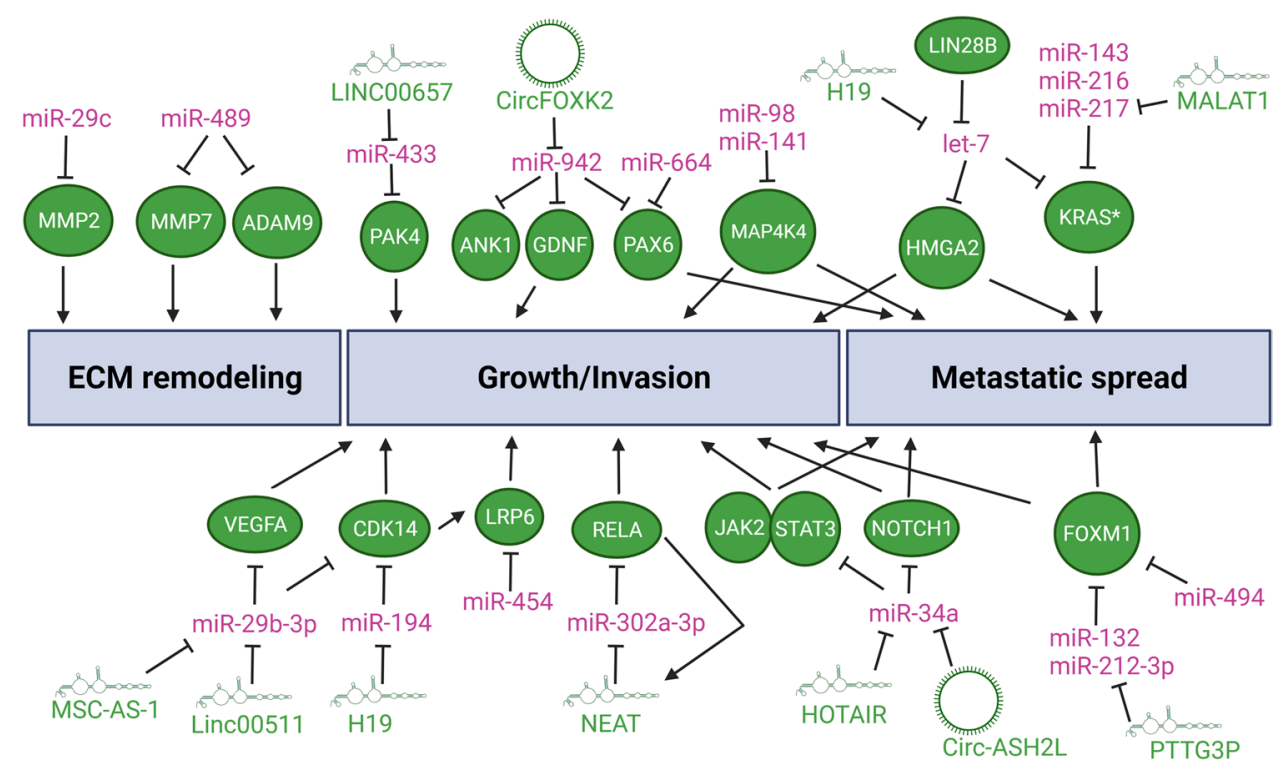

Fig. 3 miRNA-mediated regulatory networks influencing metastatic programs. miRNAs depicted in violet act as a tumor suppressive and/ or anti-metastatic factor whereas lncRNAs, circRNAs, and proteinencoding genes (ovals) in green as tumor promoting and/or pro-metastatic factors. In other contexts, these miRNAs may influence other cellular programs and decisions. Abbreviations: ADAM9, A disintegrin and metalloproteinase domain 9; ANK1, ankyrin 1; CDK14, cyclin-dependent kinase 14; FOXM1, forkhead box M1; GDNF, glial cell derived neurotrophic factor; HMGA2, high mobility group AT-Hook
2; JAK2, Janus kinase 2; KRAS*, mutant KRAS Proto-Oncogene, GTPase; LIN28B, Lin-28 homolog B; LRP6, LDL receptor related protein 6; MAP4K4, mitogen-activated protein kinase kinase kinase kinase 4; MMP2/9, matrix metallopeptidase 2/9; NOTCH1, notch receptor 1; PAK4, P21 (RAC1) activated kinase 4; PAX6, paired box 6; RELA, RELA Proto-Oncogene, NF-кB Subunit; STAT3, signal transducer and activator of transcription 3; VEGFA, vascular endothelial growth factor A 
promoting tumor growth and metastasis via EMT and immunosuppression (most of the other cases in this review) in later stages. Gene-dosage increases of mutant $K R A S$ alleles drive early stages of carcinogenesis and later metastatic spread [14]. Several miRNAs, including let-7a, miR-143, miR-216, and miR-217, directly bind to the $3^{\prime}$ UTR of both wild-type and mutant KRAS mRNAs [27, 57-65]. Overcoming this miRNA-mediated regulation of KRAS expression may also contribute to the mutant KRAS dosage-dependent switch to tumor progression and metastatic spread. let-7a, a founding member of the miRNA gene family, is considered a potent tumor suppressor gene. Other targets of let-7 include potent oncogenic factors such as c-MYC and HMGA2 [27, $64,65]$. By coordinately regulating the expression of KRAS, HMGA2, and c-MYC, let-7 activity restrains cell proliferation, cell cycle progression, invasion, EMT, and metastasis. LIN28B is an oncofetal RNA-binding protein that interferes with the maturation process of let-7 precursor RNA and in so doing increases oncogenic expression of these let-7 key target genes in PDAC $[64,65]$. LIN28B is highly expressed in circulating tumor cells of PDAC patients and is a driver of metastatic dissemination [64]. CRISPR-mediated knockout of LIN28B gene, chemical inhibition of LIN28B binding to let-7, or knockdown of HMGA2 significantly diminish the metastatic potential of circulating tumor cells [64]. This indicates the crucial role of let-7 in suppressing LIN28Bdepedent metastatic program in PDAC.

\section{Interactions between miRNAs and other non-coding RNAs dictate metastatic programs}

An emerging layer of regulation in many miRNA/key target pathways in PDAC is that of non-coding RNA dampening miRNA activity. While miRNAs are a well-defined class of short 22 nts RNAs, longer non-coding RNAs are more diverse and complex classes of RNAs (Fig. 1). Long non-coding RNAs (lncRNAs) are a heterogenous class that broadly includes a variety of non-coding RNAs longer than 200 nts [66]. IncRNAs can act as local regulators of transcription by interacting with epigenetic machinery, squelching, or blocking access of transcription factors; provide scaffolding structure for RNA-RNA and RNA-protein interactions; and bind and sequester miRNA molecules [66-68]. Circular noncoding RNAs (circRNAs) are covalently closed loops resulting from backsplicing of mRNAs $[67,69]$. The main role of circRNAs is to bind and act as sponges of miRNAs [67, 69]. We describe below a few examples of these miRNA-ncRNA interactions in regulatory pathways pertinent to tumor progression and metastatic processes already highlighted in previous sections (Figs. 2 and 3, refs [27, 35, 36, 54, 70-78]). IncRNA MALAT1 binds to miR-200c and sequesters it away from binding sites on the 3'UTR of ZEBI/ZEB2 mRNAs and other target genes, thereby skewing cellular program towards EMT [36]. Similarly, lncRNA Sox 2ot and XIST promote EMT by sequestering other miR-200 family members (miR-200a and miR-429) [35, 72]. This differential interaction and modulation of specific miR-200 family members by these lncRNA may explain, in part, discordant results of in vivo functional analysis of each family member [34]. IncRNA PCED1B-AS1 potentiates HIF-mediated EMT by sequestering miR-411-3p which directly inhibits expression of HIF1 $\alpha$ [73]. IncRNA MSCAS-1 sequesters miR-29b, a family member of EMT-regulating miR-29c, which leads to upregulation of cyclin-dependent kinase CDK14 [74]. IncRNA H19 sequesters miR-194, which also leads to upregulation of CDK14 [75]. CDK14 activates WNT signaling by phosphorylating the LRP5/6 receptors during G2/M phase of the cell cycle. Conversely, miR-454 inhibits expression of LRP6 and dampens WNT signaling [50]. This suggests a converging network of lncRNA-miRNA interactions that modulate cell cycle-dependent WNT signaling. H19 also sequesters let- $7 \mathrm{a}$ and interferes with its ability to inhibit HMGA2-mediated EMT program [27].

miR-34a is a potent tumor suppressive miRNA that can concomitantly inhibit expression of several proto-oncogenic genes (e.g., BCL2 and c-MET) and signaling pathways (e.g., NOTCH, WNT) [79]. IncRNA HOTAIR sequesters miR-34a and leads to activation of JAK2/STAT3 signaling pathway [77]. By interfering with miR-34a activity, HOTAIR promotes cancer cell stemness, EMT, and metastatic spread of PDAC cells [77]. circRNA Circ-ASH2L sequesters miR-34a what leads to upregulation of miR-34a-direct target gene NOTCH1 [76]. Circ-ASH2L-mediated activation of NOTCH signaling pathway promotes angiogenesis, tumor growth, and tumor invasion [76]. Another circRNA, CircFOXK2, also promotes tumor growth and metastasis by sequestering miR-942 and upregulating expression of its direct target genes ANK1, GDNF, and PAX6 [54]. CircFOXK2 may also influence these processes in a miR-942-independent manner by interacting with proteins involved in mRNA splicing, YBX1 and hnRNPK, which leads to upregulation of NUF2 and PDXK oncogenic expression [54]. There are additional RNA-RNA interactions of metastasis-modulating miRNAs and other ncRNA classes (e.g., IncRNA TUG1-miR-29c, lncRNA DUXAP8-miR-488) in PDAC [80-82]. Although these interactions have been reported in the context of other regulatory pathways and/or cellular processes, they may also contribute to metastatic programs. 


\section{6 microRNA-mediated cellular crosstalk influences metastatic processes}

It is well established that cancer cell-stroma interactions in PDAC have an influence on chemoresistance, disease progression, and metastatic spread. Several miRNAs have been implicated in this crosstalk between cancer cells and other cell types in the TME (Fig. 4; refs [83-88]). In some instances, miRNA-mediated regulation in cancer cells leads to a receptor/ligand-mediated response in another cell type of the TME that may then lead to a paracrine signal back to the cancer cells or other cell types in the TME. In other instances, miRNA-mediated regulation in cancer-associated fibroblasts (CAFs) or other cell types in the TME leads to physical interaction and/or paracrine signal that affects cancer cell growth and treatment resistance. miR124 is engaged in a regulatory feedback loop with NOTCH signaling pathway in cancer cells and crosstalk with tumorassociated macrophages (TAMs). miR-124 inhibits metastatic program by directly targeting JAG1 in cancer cells dampening NOTCH signaling [83]. Increased NOTCH signaling recruits and polarizes TAMs to a tumorigenic M2 phenotype [83]. IL-6 secreted by these M2 TAMs upregulates STAT3 signaling in cancer cells, which downregulates miR-124 expression and promotes EMT program [83]. Similarly, cancer cell-expressed miR-454 inhibits the expression of stromal cell derived factor 1 (SDF1, also known as chemokine CXCL12) [84]. Increased SDF1 levels recruit TAMs via activation of their chemokine receptor CXCR4, creating a pro-tumorigenic TME [84]. In contrast, cancer cell-expressed miR-128 inhibits metastatic program by directly targeting ZEB1, a positive regulator of EMT and CD47-mediated tumor immune evasion [85]. Enforced expression of miR-128 in orthotopic syngeneic Panc02 cell model dampens CD47 expression and thereby increases antitumor immunity mediated by dendritic cells, CD8 +, and Natural Killer T cells [85].

miR-21 is generally considered an oncogenic miRNA and important mediator of TGF- $\beta$-induced EMT via inhibition of PTEN, PDCD4, and/or RECK expression [89]. However, miR-21 also responds to these inputs and regulates these and other direct targets in other cellular elements of the TME, including CAFs and TAMs $[18,90]$. New 3D co-culture techniques, single-cell RNAseq, and mechanistic studies in GEMMs have uncovered the existence of distinct CAF subtypes in PDAC [91, 92]. Low or high expression of $\alpha$-smooth muscle actin (SMA) is a consistent differential feature of CAF subtypes; "myofibroblastic" CAFs (myCAFs) express high levels of SMA, are juxtaposed or in close proximity to cancer cells, and exhibit tumor-restraining function [93-99]. Co-detection of miR-21 and SMA expression demonstrates a predominant upregulation of miR-21 expression in myCAFs in the majority of PDAC tumors from patients and GEMMs [86, 87, 100-107]. myCAF expression of miR-21 provides more robust prognostic information than epithelial expression for predicting treatment response and overall survival of PDAC patients [87, 104, 105]. In vitro and in vivo coculture assays of cancer cells and fibroblasts/CAFs suggest a CAF-driven role of miR-21 [87, 104, 108]. Inhibition of miR-21 activity in CAFs before co-inoculation with cancer cells enhances efficacy of gemcitabine in a subcutaneous syngeneic Pan02 cell model [87]. Strikingly, global loss of miR-21 activity accelerates tumor development and results in a much shorter overall survival in a mutant K-Ras p53deleted PDAC GEMM (KPC model) [86]. The absence of myCAFs in precursor and invasive lesions is the most outstanding consequence of miR-21 loss. Cancer cell-specific

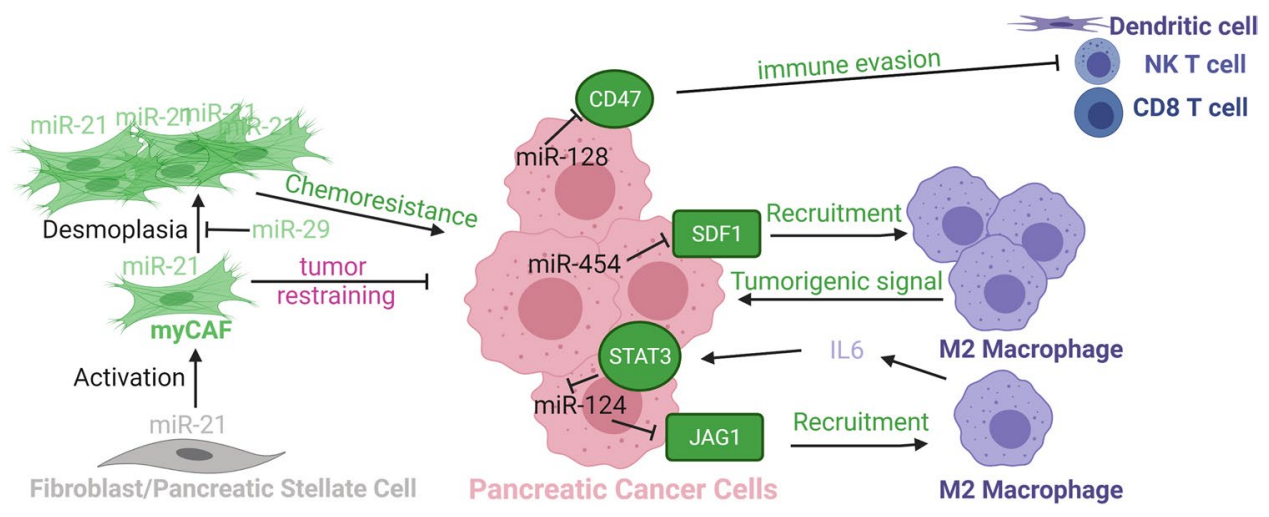

Fig. 4 miRNA-mediated cellular crosstalk influencing metastatic programs. The color of the miRNA indicates the identity of the expressing cell type. Proteins (ovals and round-edge rectangles for ligands) and processes in green depict a pro-metastatic role and process in violet an anti-metastatic role. In other contexts, these miRNAs may influence other cellular programs and decisions. Abbreviations: CD8,
T-cell surface glycoprotein CD8 alpha chain; CD47, leukocyte surface antigen CD47; IL6, interleukin 6; JAG1, jagged canonical Notch ligand 1; myCAF, myofibroblastic cancer-associated fibroblast; NK T cell, Natural Killer T cell; SDF1, stroma derived factor 1; STAT3, signal transducer and activator of transcription 3 
activity of miR-21 is not required for in vitro or in vivo growth nor affects in vitro chemoresistance to gemcitabine in this KPC model [86]. This study uncovers a pro-fibrotic yet tumor-restraining activity of miR-21 suggestive of a cell-intrinsic myCAF role. Timing, duration, and extent of miR-21 inhibition, mutational landscape, and/or tumor subtype may help explain the discrepancies between studies (Fig. 4). Interestingly, expression of miR-29a and miR-29b is downregulated in pancreatic stellate cells/resting fibroblasts during TGF- $\beta$-induced pancreatic stellate cell/CAF activation [88]. miR-29 activity appears to limit ECM protein accumulation and desmoplastic reaction via direct inhibition of collagen (COL1A1, COL3A1) and laminin (LAMC1) mRNAs [88]. Understanding how these miR-21-regulated and miR-29-regulated processes interfere with each other to affect myCAF function and composition of other CAF subtypes under chemotherapy treatment is a key question to answer in order to achieve more effective and durable clinical responses.

\section{7 microRNA-mediated pro-metastatic cell-to-cell communication via extracellular vesicles}

miRNAs themselves can be the regulatory signal from one cell type to another via transfer of extracellular vesicles (EVs). There are a few classes of membrane-bound EVs depending on their origin and size that mediate in cellto-cell communications: microparticles, shed microvesicles, or ectosomes are directly formed by budding of the cell plasma membrane and range in size $10 \mathrm{~nm}$ to $3 \mu \mathrm{m}$; exosomes are secreted via multivesicular-body endocytic process and are about $100 \mathrm{~nm}$ in size (40-150 $\mathrm{nm}$ range). Because there are technical and methodological challenges in the purification of exosomes from a mixed population of other classes of secreted vesicles [23, 109-111], we will use the more general term of EV in accordance with the International Society of Extracellular Vesicles [112] even though the original research study may have used the term exosome. We will refer to the miRNA loaded and transferred in EVs from one cell to another as Ex-miRNA. In some instance, the Ex-miRNA can act as a hormone-like signal and interact with RNA sensing receptors such as Toll-like receptor 8 [113], but generally the transferred Ex-miRNA is loaded to miRISC and controls gene expression at the posttranscriptional level in the recipient cells $[23,109,111]$. Ex-miRNAs can be secreted from cancer cells in response to treatment, hypoxia, or other inputs in the TME to affect cellular processes at a distance; recipient cells may be other cancer cells or other cell types in the TME (Fig. 5, refs [114-117]). Similarly, ex-miRNAs can be secreted from CAFs, TAMs, and other cell types in the TME to affect a variety of cellular processes in the recipient cancer cells (Fig. 5, [61, 118-125]). Ex-miR-222 secreted from more aggressive and metastasis-prone cancer cells can stimulate proliferative and invasion program in neighboring cancer cells by downregulating the expression and activity of cyclin-dependent kinase inhibitor p27 [114]. miR-222 directly binds and inhibits $p 27 \mathrm{mRNA}$ and indirectly increases AKTmediated phosphorylation and cytoplasmic translocation of p27 via inhibition of serine/threonine-protein phosphate PPP2R2A expression [114]. Radiotherapy induces the secretion of Ex-miR-194-5p from dying cancer cells [115]. Ex-miR-194-5p potentiates the survival of neighbor cancer cells, especially those with stem cell-like and tumor repopulating properties, by inducing a temporary G1/S arrest and upregulation of DNA damage response via direct inhibition of E2F3 and HMGA2 [115]. Latent release of prostaglandin E2 from dying cells stimulates proliferation of Ex-miR194-5p-protected cancer cells. Combining radiotherapy with low-dose aspirin enhances treatment efficacy by reducing both the amount of Ex-miR-194-5 pm and prostaglandin E2 released from dying cells [115]. Similarly, chemotherapy
Fig. 5 microRNA-mediated cell-to-cell communication via extracellular vesicles. The color of the Ex-miRNA indicates the identity of the cell type secreting the EV-bound miRNA. Processes in violet depict an anti-metastatic role whereas those in green a pro-metastatic role. In other contexts, these ExmiRNAs may influence other cellular programs and decisions. Abbreviations: CAF, cancerassociated fibroblast; MSC, mesenchymal stem cell; NK T cell, Natural Killer T cell

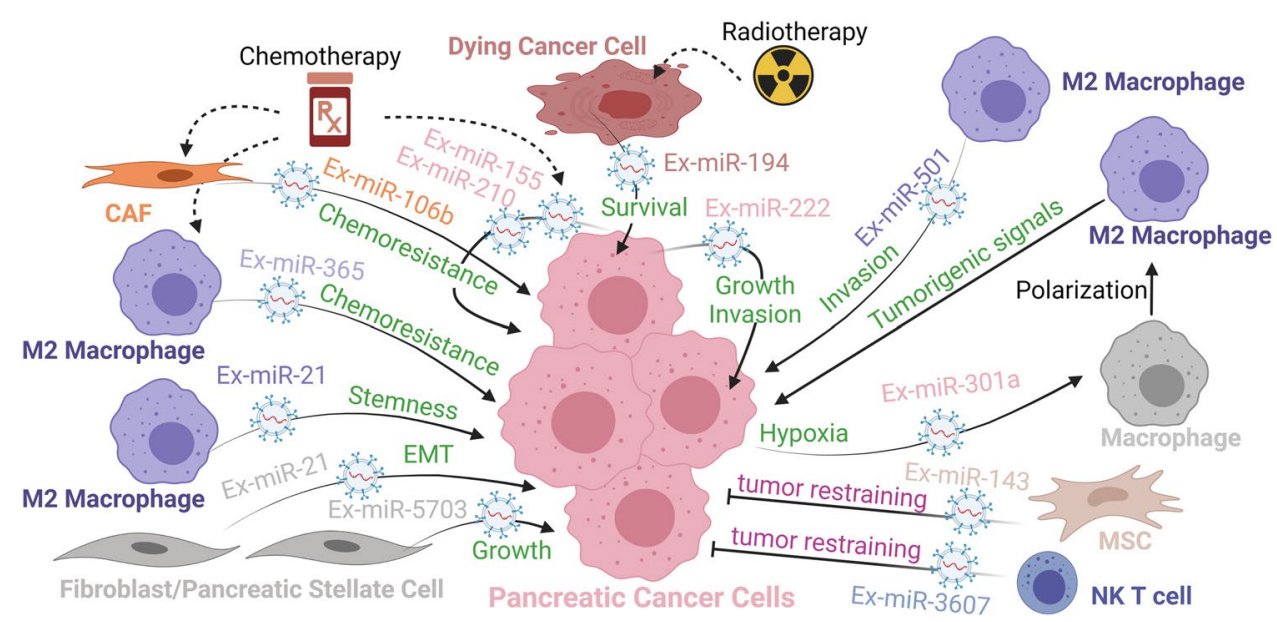


treatment with gemcitabine induces the secretion of ExmiR-155 and Ex-miR-210 from chemosensitive cancer cells, which leads to increase chemoresistance in recipient cancer cells $[116,117]$. Higher levels of miR-210 in recipient cells activate mTOR pathway, though the direct key target genes of miR-210 leading to this activation have not been identified. Gemcitabine also induces the secretion of ExmiR-106b from CAFs. In recipient cancer cells, miR-106b enhances chemoresistance via direct inhibition of TP3INPI expression [119], a stress-induced p53-target gene with antiproliferative and pro-apoptotic activity. M2-polarized TAMs secrete Ex-miR365 that contributes to gemcitabine chemoresistance in cancer cells by augmenting the gemcitabinecompeting triphospho-nucleotide pool and upregulating gemcitabine-inactivating cytidine deaminase [120]. Direct target genes that miR-365 regulates in the process have not been identified.

Hypoxic TME induces HIF-dependent release of Ex-miR301a from cancer cells to TAMs [121]. In TAMs, miR-301a upregulates $\mathrm{PI} 3 \mathrm{~K} \gamma$ signaling pathway via direct inhibition of PTEN expression, which favors polarization to tumorigenic M2 phenotype [121]. These M2 TAMs secrete TGF- $\beta$, IL-10, and arginase that promote cancer cell metastasis and potentiate miR-301a-mediated EMT program as described above (Fig. 2). While it is not clear if in direct response to Ex-miR-301a transfer, M2 TAMs can secrete Ex-miR-21 and Ex-miR-501 that are taken up by cancer cells. In cancer cells, miR-501-3p downregulates expression of TGF $\beta$ R3, whose shedded ectodomain curtails TGF- $\beta$ signaling, to favor migration and invasive behavior [122]. In cancer cells, miR-21 downregulates the expression of transcription factor KLF3 to promote NANOG/OCT4-dependent cancer cell stemness program [123]. Pancreatic stellate cells secrete Ex-miR-21 which in recipient cancer cells activates PI3K/ AKT pathway and promotes EMT program and MMP2/9mediated ECM remodeling [124]. Pancreatic stellate cells also secrete Ex-miR-5703, which in recipient cancer cells activates PI3/AKT pathway and promotes cell proliferation via downregulation of CMTM4 [125]. While beyond the scope of this review, there is growing evidence of cell-to-cell communication via secreted lncRNAs and circRNAs that inhibit miRNA activity in recipient cells in PDAC tumors $[35,126]$. This adds another exciting and complex regulatory layer to the field of RNA-RNA interactions and endogenous competing RNA hypothesis.

\section{Pharmacological interventions to modulate miRNA activity}

Several therapeutics strategies to modulate the activity of a specific miRNA with synthetic RNA analogs and different delivery technologies have been tested in preclinical models and some are being investigated in clinical trials [18, 127, 128]. An attractive feature of miRNAs as drug target is that activity modulation of a single miRNA could have a broad influence in several direct targets and processes downstream of these regulatory interactions. A potential advantage of a miRNA drug is that it could be more directed to a particular cell type and/or have a more restricted effect on selected cellular processes than small molecule inhibitors targeting upstream signaling pathways such TGF- $\beta$, JAK/STAT, or $\mathrm{PI} 3 \mathrm{~K} / \mathrm{AKT}$. There are several technical considerations for the chemical modifications and delivery approaches when replenishing the activity of tumor suppressive and/or antimetastatic miRNA vs. inhibiting the activity of a tumor promoting and/or pro-metastatic miRNA [18, 127, 128]. miRNA mimics to replenish miRNA activity are typically double-stranded modified RNA analogs that need to be taken up by cells, released in an intact form in the cytoplasm for DICER processing, miRISC loading and binding to target mRNAs, to be functionally active. In contrast, anti-miRNA inhibitors to inhibit miRNA activity are typically singlestranded modified RNA analogs that need to be taken up by cells and then released in the cytoplasm to bind to perfectly complementary miRNA molecules. Thus, the design and delivery of a miRNA mimic is more challenging than that of an anti-miRNA inhibitor $[18,128]$. miRNA mimics require encapsulation in liposomal nanoparticles or complexing with polymers or other coated nanoparticles [18, 128]. A double-stranded miR-217 mimic encapsulated in a PEGylated lipid nanoparticle, decorated with iRDG tumor penetrating-peptide, is effective at downregulating KRAS expression in vitro cell assays, but it may not be as effective as short interfering RNAs (siRNAs) against $K R A S$ in an in vivo subcutaneous PDAC tumor model [129]. miR-34a is an excellent candidate for replenishing therapy in PDAC [130]. A miR-34a-expressing DNA vector encapsulated in liposomal nanoparticles (nanovector) is effective at reducing tumor growth in subcutaneous and orthotopic xenograft models, and more so than similar nanovector strategy with KRAS-targeting miR-143 and miR-145 [130]. Forced expression of miR-34a significantly reduces tumor expression of direct target genes $(C D 44, A L D H)$ involved in cancer cell stemness [130]. miR-34 mimetic drug, MRX34, was the first miRNA replacement therapy to enter clinical trials for treatment of primary liver tumors or cases with liver metastasis [18]. Unfortunately, on-target adverse immunological effects caused termination of these clinical trials [131]. Re-formulation of the liposomal nanoparticle or use of other platforms that may preferentially deliver the miR-34 mimic to cancer cells could bypass this immune toxicity. A poly (D,L-lactide-co-glycolide) (PLGA)-based nanoparticle formulation has a high encapsulation capacity for and effectively can delivery miRNA mimics to PDAC cell lines in vitro [132]. While this nanoparticle formulation was only tested for a 
miR-150 mimic and its interaction with direct target MUC4 [132], it may be a viable and low toxicity option for systemic delivery of a miR-34 mimic. Natural or synthetic exosomes may also be used as an encapsulation and delivery vehicle to modulate miRNA activity at primary or metastatic tumor sites in PDAC [133-137]. In comparison to other nanoparticle formulation (e.g., liposomes, micelles, inorganic nanoparticles), exosomes can have a greater drug delivery potential due to their lower clearance rate, deeper tissue penetration, and enhanced biocompatibility [138, 139]. Ultrasound-assisted loading of miR-34a mimic molecules in $293 \mathrm{~T}$-derived exosomes provides a promising delivery platform to inhibit cancer cell growth in an in vivo xenograft PDAC model [140]. In this study, miR-34-loaded exosomes effectively downregulate expression of anti-apoptotic BCL2 in the xenograft tumors [140]. This or a similar strategy could be considered for investigating miR-34 replacement therapy in PDAC patients.

Anti-miRNA inhibitors can tolerate more chemical modifications since they do not require processing by the cellular machinery. Anti-miRNA inhibitors can be delivered systemically without the need of encapsulation, conjugation, or complexing, though these can offer intrinsic imaging and/or more targeted delivery capabilities. Administration of an unconjugated heavily modified single-stranded anti-miR-21 antisense oligonucleotide in the K-Ras-driven p53-mutated KPC model at an early age is remarkably effective at preventing tumor progression from pancreatic intraductal precursor lesion to malignant invasive carcinoma in this aggressive GEMM [141]. As we described above, miR-21 is a multifaceted miRNA with functional activity in different cell types. An important consideration for clinical evaluation of anti-miR-21-based cancer interception strategy will be to understand if a specific cell type or multiple ones are driving malignancy. A similar anti-miR-21 inhibitor encapsulated in PEGylated lipid nanoparticle, decorated with iRDG tumor penetrating-peptide and/or transportan cell-penetrating-peptide, reduces tumor growth in patient-derived and organoid-derived xenograft models $[129,142]$. This therapy appears to be effective only in cancer cell-derived models with high levels of miR-21 at baseline, suggesting an intertumoral heterogeneity in terms of which miR-21-expressing cell types contribute to malignancy. Expression and function of miR-10 family members, miR-10a and miR-10b, have been associated with pro-metastatic programs in PDAC [46, 143-148]. In glioblastoma and breast cancer models, several encapsulation and nanoparticle strategies, including dextran-coated iron oxide nanoparticles, have been successful at delivering anti-miR-10b modified oligonucleotides to primary and metastatic tumor sites and causing miR-10b-dependent growth inhibition [18]. A similar dextran-coated iron oxide nanoparticle platform was recently used to deliver siRNAs against immune checkpoint PD-L1 in an orthotopic Pan02 syngeneic model of PDAC [149]. Thus, this nanoparticle platform with intrinsic magnetic resonance imaging capability could be applied to investigate the therapeutic efficacy of anti-miR-10b in vivo models of PDAC.

\section{Conclusions}

The level of evidence and biological effects of these discussed miRNAs and RNA-RNA networks varies depending on experimental design, model used, and clinical validation. By design, most studies focus on a particular miRNA-mediated process, but all these miRNA-regulated processes may be occurring at once in a PDAC tumor. What the cumulative effects of these miRNAs and RNA-RNA networks are and which of these miRNA-regulated processes may have a more impactful contribution to tumor progression and metastatic spread and in what cell types are still important remaining questions. Answering these questions should guide prioritizing of clinical development of miRNA targeting strategies that would be most beneficial for PDAC patients.

Author contribution LS conceptualized and wrote manuscript, curated data, and created figures; KP edited manuscript, curated data, and edited figures; JR, AB, and TS edited manuscript and curated data. All authors reviewed and approved final version of the article.

Funding This work was supported in part by the National Cancer Institute R21 CA226579 (LS), 2020 American Society for Investigative Pathology Summer Research Opportunity Program in Pathology, and 2021 American Society for Investigative Gotlieb Undergraduate Student in Pathobiology Travel Award (KP). Figures were created with BioRender.com.

\section{Declarations}

Conflict of interest The authors declare no competing interests.

Open Access This article is licensed under a Creative Commons Attribution 4.0 International License, which permits use, sharing, adaptation, distribution and reproduction in any medium or format, as long as you give appropriate credit to the original author(s) and the source, provide a link to the Creative Commons licence, and indicate if changes were made. The images or other third party material in this article are included in the article's Creative Commons licence, unless indicated otherwise in a credit line to the material. If material is not included in the article's Creative Commons licence and your intended use is not permitted by statutory regulation or exceeds the permitted use, you will need to obtain permission directly from the copyright holder. To view a copy of this licence, visit http://creativecommons.org/licenses/by/4.0/. 


\section{References}

1. Siegel, R. L., Miller, K. D., Fuchs, H. E., \& Jemal, A. (2021). Cancer Statistics, 2021. CA: A Cancer Journal for Clinicians, 71, 7-33.

2. Mizrahi, J. D., Surana, R., Valle, J. W., \& Shroff, R. T. (2020). Pancreatic cancer. Lancet, 395, 2008-2020.

3. Kleeff, J., Korc, M., Apte, M., La Vecchia, C., Johnson, C. D., Biankin, A. V., Neale, R. E., Tempero, M., Tuveson, D. A., Hruban, R. H., \& Neoptolemos, J. P. (2016). Pancreatic cancer. Nat Rev Dis Primers, 2, 16022.

4. Conway, J. R., Herrmann, D., Evans, T. J., Morton, J. P., \& Timpson, P. (2019). Combating pancreatic cancer with PI3K pathway inhibitors in the era of personalised medicine. Gut, 68, 742-758.

5. Lafaro, K. J., \& Melstrom, L. G. (2019). The paradoxical web of pancreatic cancer tumor microenvironment. American Journal of Pathology, 189, 44-57.

6. Collisson, E. A., Bailey, P., Chang, D. K., \& Biankin, A. V. (2019). Molecular subtypes of pancreatic cancer. Nature Reviews. Gastroenterology \& Hepatology, 16, 207-220.

7. Bockorny, B., Semenisty, V., Macarulla, T., Borazanci, E., Wolpin, B. M., Stemmer, S. M., Golan, T., Geva, R., Borad, M. J., Pedersen, K. S., Park, J. O., Ramirez, R. A., Abad, D. G., Feliu, J., Munoz, A., Ponz-Sarvise, M., Peled, A., Lustig, T. M., Bohana-Kashtan, O., et al. (2020). BL-8040, a CXCR4 antagonist, in combination with pembrolizumab and chemotherapy for pancreatic cancer: The COMBAT trial. Nature Medicine, 26, 878-885.

8. Picozzi, V., Alseidi, A., Winter, J., Pishvaian, M., Mody, K., Glaspy, J., Larson, T., Matrana, M., Carney, M., Porter, S., Kouchakji, E., Rocha, F., and Carrier, E. (2020). Gemcitabine/ nab-paclitaxel with pamrevlumab: A novel drug combination and trial design for the treatment of locally advanced pancreatic cancer. ESMO Open 5.

9. Yang, J., Xu, R., Wang, C., Qiu, J., Ren, B., and You, L. (2021). Early screening and diagnosis strategies of pancreatic cancer: A comprehensive review. Cancer Commun (Lond).

10. Affolter, K. E., Hellwig, S., Nix, D. A., Bronner, M. P., Thomas, A., Fuertes, C. L., Hamil, C. L., Garrido-Laguna, I., Scaife, C. L., Mulvihill, S. J., \& Underhill, H. R. (2021). Detection of circulating tumor DNA without a tumor-informed search using nextgeneration sequencing is a prognostic biomarker in pancreatic ductal adenocarcinoma. Neoplasia, 23, 859-869.

11. Miyabayashi, K., Nakagawa, H., \& Koike, K. (2021). Molecular and phenotypic profiling for precision medicine in pancreatic cancer: Current advances and future perspectives. Front Oncol, 11,682872 .

12. Whittle, M. C., Izeradjene, K., Rani, P. G., Feng, L., Carlson, M. A., DelGiorno, K. E., Wood, L. D., Goggins, M., Hruban, R. H., Chang, A. E., Calses, P., Thorsen, S. M., \& Hingorani, S. R. (2015). RUNX3 Controls a Metastatic Switch in Pancreatic Ductal Adenocarcinoma. Cell, 161, 1345-1360.

13. Jones, S., Zhang, X., Parsons, D. W., Lin, J. C., Leary, R. J., Angenendt, P., Mankoo, P., Carter, H., Kamiyama, H., Jimeno, A., Hong, S. M., Fu, B., Lin, M. T., Calhoun, E. S., Kamiyama, M., Walter, K., Nikolskaya, T., Nikolsky, Y., Hartigan, J., et al. (2008). Core signaling pathways in human pancreatic cancers revealed by global genomic analyses. Science, 321, 1801-1806.

14. Mueller, S., Engleitner, T., Maresch, R., Zukowska, M., Lange, S., Kaltenbacher, T., Konukiewitz, B., Ollinger, R., Zwiebel, M., Strong, A., Yen, H. Y., Banerjee, R., Louzada, S., Fu, B., Seidler, B., Gotzfried, J., Schuck, K., Hassan, Z., Arbeiter, A., et al. (2018). Evolutionary routes and KRAS dosage define pancreatic cancer phenotypes. Nature, 554, 62-68.
15. Cheung, E. C., DeNicola, G. M., Nixon, C., Blyth, K., Labuschagne, C. F., Tuveson, D. A., \& Vousden, K. H. (2020). Dynamic ROS control by TIGAR regulates the initiation and progression of pancreatic cancer. Cancer Cell, 37, 168-182 e164.

16. Bartel, D. P. (2018). Metazoan MicroRNAs. Cell, 173, 20-51.

17. Gebert, L. F. R., \& MacRae, I. J. (2019). Regulation of microRNA function in animals. Nature Reviews Molecular Cell Biology, 20, 21-37.

18. Sempere, L.F., Azmi, A.S., and Moore, A. (2021). microRNAbased diagnostic and therapeutic applications in cancer medicine. Wiley Interdiscip Rev RNA, e1662.

19. Lotfi, Z., Najjary, S., Lotfi, F., Amini, M., Baghbanzadeh, A., Rashid, D. J., Asl, E. R., Baradaran, B., \& Mokhtarzadeh, A. (2021). Crosstalk between miRNAs and signaling pathways involved in pancreatic cancer and pancreatic ductal adenocarcinoma. Eur J Pharmacol, 901, 174006.

20. Rawat, M., Kadian, K., Gupta, Y., Kumar, A., Chain, P.S.G., Kovbasnjuk, O., Kumar, S., and Parasher, G. (2019). MicroRNA in pancreatic cancer: From biology to therapeutic potential. Genes (Basel) 10.

21. Tesfaye, A. A., Azmi, A. S., \& Philip, P. A. (2019). miRNA and gene expression in pancreatic ductal adenocarcinoma. American Journal of Pathology, 189, 58-70.

22. Weidle, U. H., Birzele, F., \& Nopora, A. (2019). Pancreatic ductal adenocarcinoma: MicroRNAs affecting tumor growth and metastasis in preclinical in vivo models. Cancer Genomics \& Proteomics, 16, 451-464.

23. Uddin, M.H., Al-Hallak, M.N., Philip, P.A., Mohammad, R.M., Viola, N., Wagner, K.U., and Azmi, A.S. (2021). Exosomal microRNA in pancreatic cancer diagnosis, prognosis, and treatment: From bench to bedside. Cancers (Basel) 13.

24. Fromm, B., Billipp, T., Peck, L. E., Johansen, M., Tarver, J. E., King, B. L., Newcomb, J. M., Sempere, L. F., Flatmark, K., Hovig, E., \& Peterson, K. J. (2015). A uniform system for the annotation of vertebrate microRNA genes and the evolution of the human micrornAome. Annual Review of Genetics, 49, 213-242.

25. Thomson, D. W., \& Dinger, M. E. (2016). Endogenous microRNA sponges: Evidence and controversy. Nature Reviews Genetics, 17, 272-283.

26. Rodriguez-Aznar, E., Wiesmuller, L., Sainz, B., Jr., and Hermann, P.C. (2019). EMT and stemness-key players in pancreatic cancer stem cells. Cancers (Basel) 11.

27. Ma, C., Nong, K., Zhu, H., Wang, W., Huang, X., Yuan, Z., \& $\mathrm{Ai}, \mathrm{K}$. (2014). H19 promotes pancreatic cancer metastasis by derepressing let-7's suppression on its target HMGA2-mediated EMT. Tumour Biology, 35, 9163-9169.

28. Mody, H. R., Hung, S. W., Pathak, R. K., Griffin, J., Cruz-Monserrate, Z., \& Govindarajan, R. (2017). miR-202 diminishes TGFbeta receptors and attenuates TGFbeta1-induced EMT in pancreatic cancer. Molecular Cancer Research, 15, 1029-1039.

29. Wang, C., Liu, P., Wu, H., Cui, P., Li, Y., Liu, Y., Liu, Z., \& Gou, S. (2016). MicroRNA-323-3p inhibits cell invasion and metastasis in pancreatic ductal adenocarcinoma via direct suppression of SMAD2 and SMAD3. Oncotarget, 7, 14912-14924.

30. Mody, H. R., Hung, S. W., AlSaggar, M., Griffin, J., \& Govindarajan, R. (2016). Inhibition of S-adenosylmethionine-dependent methyltransferase attenuates TGFbeta1-induced EMT and metastasis in pancreatic cancer: Putative roles of miR-663a and miR-4787-5p. Molecular Cancer Research, 14, 1124-1135.

31. Zhu, Z., Xu, Y., Zhao, J., Liu, Q., Feng, W., Fan, J., \& Wang, P. (2015). miR-367 promotes epithelial-to-mesenchymal transition and invasion of pancreatic ductal adenocarcinoma cells by targeting the Smad7-TGF-beta signalling pathway. British Journal of Cancer, 112, 1367-1375. 
32. Hu, H., Zhang, Q., Chen, W., Wu, T., Liu, S., Li, X., Luo, B., Zhang, T., Yan, G., Lu, H., \& Lu, Z. (2020). MicroRNA-301a promotes pancreatic cancer invasion and metastasis through the JAK/STAT3 signaling pathway by targeting SOCS5. Carcinogenesis, 41, 502-514.

33. Zhang, K. D., Hu, B., Cen, G., Yang, Y. H., Chen, W. W., Guo, Z. Y., Wang, X. F., Zhao, Q., \& Qiu, Z. J. (2020). MiR-301a transcriptionally activated by HIF-2alpha promotes hypoxia-induced epithelial-mesenchymal transition by targeting TP63 in pancreatic cancer. World Journal of Gastroenterology, 26, 2349-2373.

34. Diaz-Riascos, Z. V., Ginesta, M. M., Fabregat, J., Serrano, T., Busquets, J., Buscail, L., Cordelier, P., \& Capella, G. (2019). Expression and role of MicroRNAs from the miR-200 family in the tumor formation and metastatic propensity of pancreatic cancer. Mol Ther Nucleic Acids, 17, 491-503.

35. Li, Z., Jiang, P., Li, J., Peng, M., Zhao, X., Zhang, X., Chen, K., Zhang, Y., Liu, H., Gan, L., Bi, H., Zhen, P., Zhu, J., \& Li, X. (2018). Tumor-derived exosomal lnc-Sox2ot promotes EMT and stemness by acting as a ceRNA in pancreatic ductal adenocarcinoma. Oncogene, 37, 3822-3838.

36. Zhuo, M., Yuan, C., Han, T., Cui, J., Jiao, F., \& Wang, L. (2018). A novel feedback loop between high MALAT-1 and low miR$200 c-3 p$ promotes cell migration and invasion in pancreatic ductal adenocarcinoma and is predictive of poor prognosis. $B M C$ Cancer, 18, 1032.

37. Zhao, G., Wang, B., Liu, Y., Zhang, J. G., Deng, S. C., Qin, Q., Tian, K., Li, X., Zhu, S., Niu, Y., Gong, Q., \& Wang, C. Y. (2013). miRNA-141, downregulated in pancreatic cancer, inhibits cell proliferation and invasion by directly targeting MAP4K4. Molecular Cancer Therapeutics, 12, 2569-2580.

38. Pan, Y., Lu, F., Xiong, P., Pan, M., Zhang, Z., Lin, X., Pan, M., \& Huang, H. (2018). WIPF1 antagonizes the tumor suppressive effect of miR-141/200c and is associated with poor survival in patients with PDAC. Journal of Experimental \& Clinical Cancer Research, 37, 167.

39. Zhang, Z., Li, J., Guo, H., Wang, F., Ma, L., Du, C., Wang, Y., Wang, Q., Kornmann, M., Tian, X., \& Yang, Y. (2019). BRM transcriptionally regulates miR-302a-3p to target SOCS5/STAT3 signaling axis to potentiate pancreatic cancer metastasis. Cancer Letters, 449, 215-225.

40. Yu, D. L., Zhang, T., Wu, K., Li, Y., Wang, J., Chen, J., Li, X. Q., Peng, X. G., Wang, J. N., \& Tan, L. G. (2017). MicroRNA-448 suppresses metastasis of pancreatic ductal adenocarcinoma through targeting JAK1/STAT3 pathway. Oncology Reports, 38, 1075-1082.

41. Xiong, Y., Wang, Y., Wang, L., Huang, Y., Xu, Y., Xu, L., Guo, Y., Lu, J., Li, X., Zhu, M., \& Qian, H. (2018). MicroRNA-30b targets snail to impede epithelial-mesenchymal transition in pancreatic cancer stem cells. Journal of Cancer, 9, 2147-2159.

42. Xiong, J., Wang, D., Wei, A., Lu, H., Tan, C., Li, A., Tang, J., Wang, Y., He, S., Liu, X., \& Hu, W. (2017). Deregulated expression of miR-107 inhibits metastasis of PDAC through inhibition PI3K/Akt signaling via caveolin-1 and PTEN. Experimental Cell Research, 361, 316-323.

43. Hu, J., Li, L., Chen, H., Zhang, G., Liu, H., Kong, R., Chen, H., Wang, Y., Li, Y., Tian, F., Lv, X., Li, G., \& Sun, B. (2018). MiR-361-3p regulates ERK1/2-induced EMT via DUSP2 mRNA degradation in pancreatic ductal adenocarcinoma. Cell Death \& Disease, 9, 807.

44. Ma, G., Li, G., Fan, W., Xu, Y., Song, S., Guo, K., \& Liu, Z. (2021). Circ-0005105 activates COL11A1 by targeting miR$20 \mathrm{a}-3 \mathrm{p}$ to promote pancreatic ductal adenocarcinoma progression. Cell Death \& Disease, 12, 656.

45. Ma, J., Zhang, J., Weng, Y. C., \& Wang, J. C. (2018). EZH2mediated microRNA-139-5p regulates epithelial-mesenchymal transition and lymph node metastasis of pancreatic cancer. $\mathrm{Mol}$ ecules and Cells, 41, 868-880.

46. Wang, C., Yin, W., \& Liu, H. (2020). MicroRNA-10a promotes epithelial-to-mesenchymal transition and stemness maintenance of pancreatic cancer stem cells via upregulating the Hippo signaling pathway through WWC2 inhibition. Journal of Cellular Biochemistry, 121, 4505-4521.

47. Kai, F., Drain, A. P., \& Weaver, V. M. (2019). The extracellular matrix modulates the metastatic journey. Developmental Cell, 49, 332-346.

48. Zou, Y., Li, J., Chen, Z., Li, X., Zheng, S., Yi, D., Zhong, A., \& Chen, J. (2015). miR-29c suppresses pancreatic cancer liver metastasis in an orthotopic implantation model in nude mice and affects survival in pancreatic cancer patients. Carcinogenesis, 36, 676-684.

49. Yuan, P., He, X. H., Rong, Y. F., Cao, J., Li, Y., Hu, Y. P., Liu, Y., Li, D., Lou, W., \& Liu, M. F. (2017). KRAS/NF-kappaB/YY1/ miR-489 signaling axis controls pancreatic cancer metastasis. Cancer Research, 77, 100-111.

50. Fan, Y., Shi, C., Li, T., \& Kuang, T. (2017). microRNA-454 shows anti-angiogenic and anti-metastatic activity in pancreatic ductal adenocarcinoma by targeting LRP6. American Journal of Cancer Research, 7, 139-147.

51. Fu, Y., Liu, X., Chen, Q., Liu, T., Lu, C., Yu, J., Miao, Y., \& Wei, J. (2018). Downregulated miR-98-5p promotes PDAC proliferation and metastasis by reversely regulating MAP4K4. Journal of Experimental \& Clinical Cancer Research, 37, 130.

52. Zhu, D., Zheng, S., Fang, C., Guo, X., Han, D., Tang, M., Fu, H., Jiang, M., Xie, N., Nie, Y., Yao, X., \& Chen, Y. (2020). Dysbindin promotes pancreatic ductal adenocarcinoma metastasis by activating NF-kappaB/MDM2 via miR-342-3p. Cancer Letters, 477, 107-121.

53. Wang, Q., Wang, J., Niu, S., Wang, S., Liu, Y., \& Wang, X. (2019). MicroRNA-664 targets paired box protein 6 to inhibit the oncogenicity of pancreatic ductal adenocarcinoma. International Journal of Oncology, 54, 1884-1896.

54. Wong, C. H., Lou, U. K., Li, Y., Chan, S. L., Tong, J. H., To, K. F., \& Chen, Y. (2020). CircFOXK2 promotes growth and metastasis of pancreatic ductal adenocarcinoma by complexing with RNA-binding proteins and sponging MiR-942. Cancer Research, 80, 2138-2149.

55. Liu, W., Tang, J., Zhang, H., Kong, F., Zhu, H., Li, P., Li, Z., Kong, X., \& Wang, K. (2020). A novel lncRNA PTTG3P/miR132/212-3p/FoxM1 feedback loop facilitates tumorigenesis and metastasis of pancreatic cancer. Cell Death Discov, 6, 136.

56. Li, L., Li, Z., Kong, X., Xie, D., Jia, Z., Jiang, W., Cui, J., Du, Y., Wei, D., Huang, S., \& Xie, K. (2014). Down-regulation of microRNA-494 via loss of SMAD4 increases FOXM1 and betacatenin signaling in pancreatic ductal adenocarcinoma cells. Gastroenterology, 147, 485-497 e418.

57. Azevedo-Pouly, A. C., Sutaria, D. S., Jiang, J., Elgamal, O. A., Amari, F., Allard, D., Grippo, P. J., Coppola, V., \& Schmittgen, T. D. (2017). miR-216 and miR-217 expression is reduced in transgenic mouse models of pancreatic adenocarcinoma, knockout of miR-216/miR-217 host gene is embryonic lethal. Functional \& Integrative Genomics, 17, 203-212.

58. Liu, P., Yang, H., Zhang, J., Peng, X., Lu, Z., Tong, W., \& Chen, J. (2017). The lncRNA MALAT1 acts as a competing endogenous RNA to regulate KRAS expression by sponging miR-217 in pancreatic ductal adenocarcinoma. Science and Reports, 7, 5186.

59. Sutaria, D. S., Jiang, J., Azevedo-Pouly, A. C., Wright, L., Bray, J. A., Fredenburg, K., Liu, X., Lu, J., Torres, C., Mancinelli, G., Grippo, P. J., Coppola, V., \& Schmittgen, T. D. (2019). Knockout 
of acinar enriched microRNAs in mice promote duct formation but not pancreatic cancer. Science and Reports, 9, 11147.

60. Zhao, W. G., Yu, S. N., Lu, Z. H., Ma, Y. H., Gu, Y. M., \& Chen, J. (2010). The miR-217 microRNA functions as a potential tumor suppressor in pancreatic ductal adenocarcinoma by targeting KRAS. Carcinogenesis, 31, 1726-1733.

61. Wang, B., Xu, Y., Wei, Y., Lv, L., Liu, N., Lin, R., Wang, X., \& Shi, B. (2021). Human mesenchymal stem cell-derived exosomal microRNA-143 promotes apoptosis and suppresses cell growth in pancreatic cancer via target gene regulation. Front Genet, 12, 581694.

62. Xie, F., Li, C., Zhang, X., Peng, W., \& Wen, T. (2019). MiR143-3p suppresses tumorigenesis in pancreatic ductal adenocarcinoma by targeting KRAS. Biomed Pharmacother, 119, 109424.

63. Hu, Y., Ou, Y., Wu, K., Chen, Y., \& Sun, W. (2012). miR-143 inhibits the metastasis of pancreatic cancer and an associated signaling pathway. Tumour Biology, 33, 1863-1870.

64. Franses, J. W., Philipp, J., Missios, P., Bhan, I., Liu, A., Yashaswini, C., Tai, E., Zhu, H., Ligorio, M., Nicholson, B., Tassoni, E. M., Desai, N., Kulkarni, A. S., Szabolcs, A., Hong, T. S., Liss, A. S., Fernandez-Del Castillo, C., Ryan, D. P., Maheswaran, S., et al. (2020). Pancreatic circulating tumor cell profiling identifies LIN28B as a metastasis driver and drug target. Nature Cоттиnications, 11, 3303.

65. Wang, Y., Li, J., Guo, S., Ouyang, Y., Yin, L., Liu, S., Zhao, Z., Yang, J., Huang, W., Qin, H., Zhao, X., Ni, B., \& Wang, H. (2017). Lin28B facilitates the progression and metastasis of pancreatic ductal adenocarcinoma. Oncotarget, 8, 60414-60428.

66. Statello, L., Guo, C. J., Chen, L. L., \& Huarte, M. (2021). Gene regulation by long non-coding RNAs and its biological functions. Nature Reviews Molecular Cell Biology, 22, 96-118.

67. Anfossi, S., Babayan, A., Pantel, K., \& Calin, G. A. (2018). Clinical utility of circulating non-coding RNAs - An update. Nature Reviews. Clinical Oncology, 15, 541-563.

68. Machiela, E., Popkie, A., and Sempere, L. (2015). Individual noncoding RNA variations: Their role in shaping and maintaining the epigenetic landscape. In Personalized Epigenetics, Trygve Tollefsbol, ed. Waltham, Massachusetts: Academic Press, 2015, Chapter 4 pages: 84-114. ISBN-13: 978-0124201354.

69. Kristensen, L. S., Andersen, M. S., Stagsted, L. V. W., Ebbesen, K. K., Hansen, T. B., \& Kjems, J. (2019). The biogenesis, biology and characterization of circular RNAs. Nature Reviews Genetics, 20, 675-691.

70. Zhao, X., Liu, Y., Li, Z., Zheng, S., Wang, Z., Li, W., Bi, Z., Li, L., Jiang, Y., Luo, Y., Lin, Q., Fu, Z., \& Rufu, C. (2018). Linc00511 acts as a competing endogenous RNA to regulate VEGFA expression through sponging hsa-miR-29b-3p in pancreatic ductal adenocarcinoma. Journal of Cellular and Molecular Medicine, 22, 655-667.

71. Luo, Z., Yi, Z. J., Ou, Z. L., Han, T., Wan, T., Tang, Y. C., Wang, Z. C., \& Huang, F. Z. (2019). RELA/NEAT1/miR-302a-3p/ RELA feedback loop modulates pancreatic ductal adenocarcinoma cell proliferation and migration. Journal of Cellular Physiology, 234, 3583-3597.

72. Shen, J., Hong, L., Yu, D., Cao, T., Zhou, Z., \& He, S. (2019). LncRNA XIST promotes pancreatic cancer migration, invasion and EMT by sponging miR-429 to modulate ZEB1 expression. International Journal of Biochemistry \& Cell Biology, 113, $17-26$.

73. Zhang, Y., Ma, H., and Chen, C. (2021). Long noncoding RNA PCED1BAS1 promotes pancreatic ductal adenocarcinoma progression by regulating the miR4113p/HIF1alpha axis. Oncol Rep 46.

74. Sun, Y., Wang, P., Yang, W., Shan, Y., Zhang, Q., \& Wu, H. (2019). The role of lncRNA MSC-AS1/miR-29b-3p axis-mediated CDK14 modulation in pancreatic cancer proliferation and Gemcitabine-induced apoptosis. Cancer Biology \& Therapy, 20, 729-739.

75. Sun, Y., Zhu, Q., Yang, W., Shan, Y., Yu, Z., Zhang, Q., \& Wu, H. (2019). LncRNA H19/miR-194/PFTK1 axis modulates the cell proliferation and migration of pancreatic cancer. Journal of Cellular Biochemistry, 120, 3874-3886.

76. Chen, Y., Li, Z., Zhang, M., Wang, B., Ye, J., Zhang, Y., Tang, D., Ma, D., Jin, W., Li, X., \& Wang, S. (2019). Circ-ASH2L promotes tumor progression by sponging miR-34a to regulate Notch1 in pancreatic ductal adenocarcinoma. Journal of Experimental \& Clinical Cancer Research, 38, 466.

77. Deng, S., Wang, J., Zhang, L., Li, J., \& Jin, Y. (2021). LncRNA HOTAIR promotes cancer stem-like cells properties by sponging miR-34a to activate the JAK2/STAT3 pathway in pancreatic ductal adenocarcinoma. Oncotargets and Therapy, 14, 1883-1893.

78. Bi, S., Wang, Y., Feng, H., \& Li, Q. (2020). Long noncoding RNA LINC00657 enhances the malignancy of pancreatic ductal adenocarcinoma by acting as a competing endogenous RNA on microRNA-433 to increase PAK4 expression. Cell Cycle, 19, 801-816.

79. Li, W. J., Wang, Y., Liu, R., Kasinski, A. L., Shen, H., Slack, F. J., \& Tang, D. G. (2021). MicroRNA-34a: potent tumor suppressor, cancer stem cell inhibitor, and potential anticancer therapeutic. Front Cell Dev Biol, 9, 640587.

80. Lu, Y., Tang, L., Zhang, Z., Li, S., Liang, S., Ji, L., Yang, B., Liu, Y., \& Wei, W. (2018). Long noncoding RNA TUG1/miR-29c axis affects cell proliferation, invasion, and migration in human pancreatic cancer. Disease Markers, 2018, 6857042.

81. Li, J. R., Liu, L., Luo, H., Chen, Z. G., Wang, J. H., \& Li, N. F. (2021). Long noncoding RNA DUXAP8 promotes pancreatic carcinoma cell migration and invasion via pathway by miR-448/ WTAP/Fak signaling axis. Pancreas, 50, 317-326.

82. Xiong, G., Liu, C., Yang, G., Feng, M., Xu, J., Zhao, F., You, L., Zhou, L., Zheng, L., Hu, Y., Wang, X., Zhang, T., \& Zhao, Y. (2019). Long noncoding RNA GSTM3TV2 upregulates LAT2 and OLR1 by competitively sponging let-7 to promote gemcitabine resistance in pancreatic cancer. Journal of Hematology \& Oncology, 12, 97.

83. Geng, Y., Fan, J., Chen, L., Zhang, C., Qu, C., Qian, L., Chen, K., Meng, Z., Chen, Z., \& Wang, P. (2021). A Notch-dependent inflammatory feedback circuit between macrophages and cancer cells regulates pancreatic cancer metastasis. Cancer Research, 81, 64-76.

84. Fan, Y., Xu, L. L., Shi, C. Y., Wei, W., Wang, D. S., \& Cai, D. F. (2016). MicroRNA-454 regulates stromal cell derived factor-1 in the control of the growth of pancreatic ductal adenocarcinoma. Science and Reports, 6, 22793.

85. Xi, Q., Chen, Y., Yang, G. Z., Zhang, J. Y., Zhang, L. J., Guo, X. D., Zhao, J. Y., Xue, Z. Y., Li, Y., \& Zhang, R. (2020). miR-128 regulates tumor cell CD47 expression and promotes anti-tumor immunity in pancreatic cancer. Frontiers in Immunology, 11, 890.

86. Schipper, J., Westerhuis, J. J., Beddows, I., Madaj, Z., Monsma, D., Hostetter, G., Kiupel, M., Conejo-Garcia, J. R., \& Sempere, L. F. (2020). Loss of microRNA-21 leads to profound stromal remodeling and short survival in K-Ras-driven mouse models of pancreatic cancer. International Journal of Cancer, 147, 2265-2278.

87. Zhang, L., Yao, J., Li, W., \& Zhang, C. (2018). Micro-RNA-21 regulates cancer-associated fibroblast-mediated drug resistance in pancreatic cancer. Oncology Research, 26, 827-835.

88. Kwon, J. J., Nabinger, S. C., Vega, Z., Sahu, S. S., Alluri, R. K., Abdul-Sater, Z., Yu, Z., Gore, J., Nalepa, G., Saxena, R., Korc, 
M., \& Kota, J. (2015). Pathophysiological role of microRNA-29 in pancreatic cancer stroma. Science and Reports, 5, 11450.

89. Bautista-Sanchez, D., Arriaga-Canon, C., Pedroza-Torres, A., De La Rosa-Velazquez, I. A., Gonzalez-Barrios, R., ContrerasEspinosa, L., Montiel-Manriquez, R., Castro-Hernandez, C., Fragoso-Ontiveros, V., Alvarez-Gomez, R. M., \& Herrera, L. A. (2020). The promising role of miR-21 as a cancer biomarker and its importance in RNA-based therapeutics. Mol Ther Nucleic Acids, 20, 409-420.

90. Sheedy, F. J. (2015). Turning 21: Induction of miR-21 as a key switch in the inflammatory response. Frontiers in Immunology, $6,19$.

91. Biffi, G., \& Tuveson, D. A. (2021). Diversity and biology of cancer-associated fibroblasts. Physiological Reviews, 101, 147-176.

92. Helms, E., Onate, M. K., \& Sherman, M. H. (2020). Fibroblast heterogeneity in the pancreatic tumor microenvironment. Cancer Discovery, 10, 648-656.

93. Ohlund, D., Handly-Santana, A., Biffi, G., Elyada, E., Almeida, A. S., Ponz-Sarvise, M., Corbo, V., Oni, T. E., Hearn, S. A., Lee, E. J., Chio, I. I., Hwang, C. I., Tiriac, H., Baker, L. A., Engle, D. D., Feig, C., Kultti, A., Egeblad, M., Fearon, D. T., et al. (2017). Distinct populations of inflammatory fibroblasts and myofibroblasts in pancreatic cancer. Journal of Experimental Medicine, 214, 579-596.

94. Biffi, G., Oni, T. E., Spielman, B., Hao, Y., Elyada, E., Park, Y., Preall, J., \& Tuveson, D. A. (2019). IL1-induced JAK/STAT signaling is antagonized by TGFbeta to shape CAF Heterogeneity in pancreatic ductal adenocarcinoma. Cancer Discovery, 9, 282-301.

95. Elyada, E., Bolisetty, M., Laise, P., Flynn, W. F., Courtois, E. T., Burkhart, R. A., Teinor, J. A., Belleau, P., Biffi, G., Lucito, M. S., Sivajothi, S., Armstrong, T. D., Engle, D. D., Yu, K. H., Hao, Y., Wolfgang, C. L., Park, Y., Preall, J., Jaffee, E. M., et al. (2019). Cross-species single-cell analysis of pancreatic ductal adenocarcinoma reveals antigen-presenting cancer-associated fibroblasts. Cancer Discovery, 9, 1102-1123.

96. Steele, N.G., Biffi, G., Kemp, S.B., Zhang, Y., Drouillard, D., Syu, L., Hao, Y., Oni, T.E., Brosnan, E., Elyada, E., Doshi, A., Hansma, C., Espinoza, C., Abbas, A., The, S., Irizarry-Negron, V., Halbrook, C.J., Franks, N.E., Hoffman, M.T., et al. (2021). Inhibition of hedgehog signaling alters fibroblast composition in pancreatic cancer. Clin Cancer Res.

97. Ozdemir, B. C., Pentcheva-Hoang, T., Carstens, J. L., Zheng, X., Wu, C. C., Simpson, T. R., Laklai, H., Sugimoto, H., Kahlert, C., Novitskiy, S. V., De Jesus-Acosta, A., Sharma, P., Heidari, P., Mahmood, U., Chin, L., Moses, H. L., Weaver, V. M., Maitra, A., Allison, J. P., et al. (2014). Depletion of carcinoma-associated fibroblasts and fibrosis induces immunosuppression and accelerates pancreas cancer with reduced survival. Cancer Cell, 25, 719-734.

98. Rhim, A. D., Oberstein, P. E., Thomas, D. H., Mirek, E. T., Palermo, C. F., Sastra, S. A., Dekleva, E. N., Saunders, T., Becerra, C. P., Tattersall, I. W., Westphalen, C. B., Kitajewski, J., Fernandez-Barrena, M. G., Fernandez-Zapico, M. E., Iacobuzio-Donahue, C., Olive, K. P., \& Stanger, B. Z. (2014). Stromal elements act to restrain, rather than support, pancreatic ductal adenocarcinoma. Cancer Cell, 25, 735-747.

99. Lee, J. J., Perera, R. M., Wang, H., Wu, D. C., Liu, X. S., Han, S., Fitamant, J., Jones, P. D., Ghanta, K. S., Kawano, S., Nagle, J. M., Deshpande, V., Boucher, Y., Kato, T., Chen, J. K., Willmann, J. K., Bardeesy, N., \& Beachy, P. A. (2014). Stromal response to Hedgehog signaling restrains pancreatic cancer progression. Proc Natl Acad Sci U S A, 111, E3091-3100.

100. Sempere, L. F., Preis, M., Yezefski, T., Ouyang, H., Suriawinata, A. A., Silahtaroglu, A., Conejo-Garcia, J. R., Kauppinen, S., Wells, W., \& Korc, M. (2010). Fluorescence-based codetection with protein markers reveals distinct cellular compartments for altered MicroRNA expression in solid tumors. Clinical Cancer Research, 16, 4246-4255.

101. Preis, M., Gardner, T.B., Gordon, S.R., Pipas, M.J., Mackenzie, T.A., Klein, E.E., Longnecker, D.S., Gutmann, E.J., Sempere, L.F., and Korc, M. (2011). microRNA-10b expression correlates with response to neoadjuvant therapy and survival in pancreatic ductal adenocarcinoma. Clin. Cancer Res.

102. Sempere, L. F., \& Korc, M. (2013). A method for conducting highly sensitive microRNA in situ hybridization and immunohistochemical analysis in pancreatic cancer. Methods in Molecular Biology, 980, 43-59.

103. Sempere, L. F., Zaluzec, E., Kenyon, E., Kiupel, M., \& Moore, A. (2020). Automated five-color multiplex co-detection of Microrna and protein expression in fixed tissue specimens. Methods in Molecular Biology, 2148, 257-276.

104. Kadera, B. E., Li, L., Toste, P. A., Wu, N., Adams, C., Dawson, D. W., \& Donahue, T. R. (2013). MicroRNA-21 in pancreatic ductal adenocarcinoma tumor-associated fibroblasts promotes metastasis. PLoS ONE, 8, e71978.

105. Donahue, T. R., Nguyen, A. H., Moughan, J., Li, L., Tatishchev, S., Toste, P., \& Farrell, J. J. (2014). Stromal microRNA-21 levels predict response to 5 -fluorouracil in patients with pancreatic cancer. Journal of Surgical Oncology, 110, 952-959.

106. Ensink, E., Sinha, J., Sinha, A., Tang, H., Calderone, H. M., Hostetter, G., Winter, J., Cherba, D., Brand, R. E., Allen, P. J., Sempere, L. F., \& Haab, B. B. (2015). Segment and fit thresholding: A new method for image analysis applied to microarray and immunofluorescence data. Analytical Chemistry, 87, 9715-9721.

107. du Rieu, M. C., Torrisani, J., Selves, J., Al, S. T., Souque, A., Dufresne, M., Tsongalis, G. J., Suriawinata, A. A., Carrere, N., Buscail, L., \& Cordelier, P. (2010). MicroRNA-21 is induced early in pancreatic ductal adenocarcinoma precursor lesions. Clinical Chemistry, 56, 603-612.

108. Chen, S., Chen, X., Shan, T., Ma, J., Lin, W., Li, W., \& Kang, Y. (2018). MiR-21-mediated metabolic alteration of cancerassociated fibroblasts and its effect on pancreatic cancer cell behavior. International Journal of Biological Sciences, 14, 100-110.

109. Sempere, L.F., Keto, J., and Fabbri, M. (2017). Exosomal MicroRNAs in breast cancer towards diagnostic and therapeutic applications. Cancers (Basel) 9.

110. Kalluri, R. (2016). The biology and function of exosomes in cancer. The Journal of Clinical Investigation, 126, 1208-1215.

111. Hussain, Z., Nigri, J., and Tomasini, R. (2021). The cellular and biological impact of extracellular vesicles in pancreatic cancer. Cancers (Basel) 13.

112. Thery, C., Witwer, K. W., Aikawa, E., Alcaraz, M. J., Anderson, J. D., Andriantsitohaina, R., Antoniou, A., Arab, T., Archer, F., Atkin-Smith, G. K., Ayre, D. C., Bach, J. M., Bachurski, D., Baharvand, H., Balaj, L., Baldacchino, S., Bauer, N. N., Baxter, A. A., Bebawy, M., et al. (2018). Minimal information for studies of extracellular vesicles 2018 (MISEV2018): A position statement of the International Society for Extracellular Vesicles and update of the MISEV2014 guidelines. J Extracell Vesicles, 7, 1535750.

113. Pardini, B., and Calin, G.A. (2019). MicroRNAs and long noncoding RNAs and their hormone-like activities in cancer. Cancers (Basel) 11.

114. Li, Z., Tao, Y., Wang, X., Jiang, P., Li, J., Peng, M., Zhang, X., Chen, K., Liu, H., Zhen, P., Zhu, J., Liu, X., \& Liu, X. (2018). Tumor-secreted exosomal miR-222 promotes tumor progression via regulating P27 Expression and re-localization in pancreatic cancer. Cellular Physiology and Biochemistry, 51, 610-629. 
115. Jiang, M. J., Chen, Y. Y., Dai, J. J., Gu, D. N., Mei, Z., Liu, F. R., Huang, Q., \& Tian, L. (2020). Dying tumor cell-derived exosomal miR-194-5p potentiates survival and repopulation of tumor repopulating cells upon radiotherapy in pancreatic cancer. Molecular Cancer, 19, 68.

116. Yang, Z., Zhao, N., Cui, J., Wu, H., Xiong, J., \& Peng, T. (2020). Exosomes derived from cancer stem cells of gemcitabine-resistant pancreatic cancer cells enhance drug resistance by delivering miR-210. Cellular Oncology (Dordrecht), 43, 123-136.

117. Mikamori, M., Yamada, D., Eguchi, H., Hasegawa, S., Kishimoto, T., Tomimaru, Y., Asaoka, T., Noda, T., Wada, H., Kawamoto, K., Gotoh, K., Takeda, Y., Tanemura, M., Mori, M., \& Doki, Y. (2017). MicroRNA-155 controls exosome synthesis and promotes gemcitabine resistance in pancreatic ductal adenocarcinoma. Science and Reports, 7, 42339.

118. Sun, H., Shi, K., Qi, K., Kong, H., Zhang, J., Dai, S., Ye, W., Deng, T., He, Q., \& Zhou, M. (2019). Natural Killer cell-derived exosomal miR-3607-3p inhibits pancreatic cancer progression by targeting IL-26. Frontiers in Immunology, 10, 2819.

119. Fang, Y., Zhou, W., Rong, Y., Kuang, T., Xu, X., Wu, W., Wang, D., \& Lou, W. (2019). Exosomal miRNA-106b from cancer-associated fibroblast promotes gemcitabine resistance in pancreatic cancer. Exp Cell Res, 383, 111543.

120. Binenbaum, Y., Fridman, E., Yaari, Z., Milman, N., Schroeder, A., Ben David, G., Shlomi, T., \& Gil, Z. (2018). Transfer of miRNA in macrophage-derived exosomes induces drug resistance in pancreatic adenocarcinoma. Cancer Research, 78, $5287-5299$.

121. Wang, X., Luo, G., Zhang, K., Cao, J., Huang, C., Jiang, T., Liu, B., Su, L., \& Qiu, Z. (2018). Hypoxic tumor-derived exosomal miR-301a mediates M2 macrophage polarization via PTEN/PI3Kgamma to promote pancreatic cancer metastasis. Cancer Research, $78,4586-4598$

122. Yin, Z., Ma, T., Huang, B., Lin, L., Zhou, Y., Yan, J., Zou, Y., \& Chen, S. (2019). Macrophage-derived exosomal microRNA-501-3p promotes progression of pancreatic ductal adenocarcinoma through the TGFBR3-mediated TGF-beta signaling pathway. Journal of Experimental \& Clinical Cancer Research, 38, 310.

123. Chang, J., Li, H., Zhu, Z., Mei, P., Hu, W., Xiong, X., and Tao, J. (2021). microRNA-21-5p from M2 macrophage-derived extracellular vesicles promotes the differentiation and activity of pancreatic cancer stem cells by mediating KLF3. Cell Biol Toxicol.

124. Ma, Q., Wu, H., Xiao, Y., Liang, Z., \& Liu, T. (2020). Upregulation of exosomal microRNA21 in pancreatic stellate cells promotes pancreatic cancer cell migration and enhances Ras/ERK pathway activity. International Journal of Oncology, 56, 1025-1033.

125. Li, M., Guo, H., Wang, Q., Chen, K., Marko, K., Tian, X., \& Yang, Y. (2020). Pancreatic stellate cells derived exosomal miR-5703 promotes pancreatic cancer by downregulating CMTM4 and activating PI3K/Akt pathway. Cancer Letters, 490, 20-30.

126. Li, Z., Yanfang, W., Li, J., Jiang, P., Peng, T., Chen, K., Zhao, X., Zhang, Y., Zhen, P., Zhu, J., \& Li, X. (2018). Tumor-released exosomal circular RNA PDE8A promotes invasive growth via the miR-338/MACC1/MET pathway in pancreatic cancer. Cancer Letters, 432, 237-250.

127. Roberts, T. C., Langer, R., \& Wood, M. J. A. (2020). Advances in oligonucleotide drug delivery. Nature Reviews. Drug Discovery, 19, 673-694.

128. Forterre, A., Komuro, H., Aminova, S., and Harada, M. (2020). A comprehensive review of cancer MicroRNA therapeutic delivery strategies. Cancers (Basel) 12.

129. Gilles, M. E., Hao, L., Brown, K., Lim, J., Bhatia, S. N., \& Slack, F. J. (2019). Tumor penetrating nanomedicine targeting both an oncomiR and an oncogene in pancreatic cancer. Oncotarget, 10, 5349-5358.
130. Pramanik, D., Campbell, N. R., Karikari, C., Chivukula, R., Kent, O. A., Mendell, J. T., \& Maitra, A. (2011). Restitution of tumor suppressor microRNAs using a systemic nanovector inhibits pancreatic cancer growth in mice. Molecular Cancer Therapeutics, 10, $1470-1480$

131. Hong, D. S., Kang, Y. K., Borad, M., Sachdev, J., Ejadi, S., Lim, H. Y., Brenner, A. J., Park, K., Lee, J. L., Kim, T. Y., Shin, S., Becerra, C. R., Falchook, G., Stoudemire, J., Martin, D., Kelnar, K., Peltier, H., Bonato, V., Bader, A. G., et al. (2020). Phase 1 study of MRX34, a liposomal miR-34a mimic, in patients with advanced solid tumours. British Journal of Cancer, 122, 1630-1637.

132. Arora, S., Swaminathan, S. K., Kirtane, A., Srivastava, S. K., Bhardwaj, A., Singh, S., Panyam, J., \& Singh, A. P. (2014). Synthesis, characterization, and evaluation of poly (D, L-lactideco-glycolide)-based nanoformulation of miRNA-150: Potential implications for pancreatic cancer therapy. International Journal of Nanomedicine, 9, 2933-2942.

133. Ding, Y., Cao, F., Sun, H., Wang, Y., Liu, S., Wu, Y., Cui, Q., Mei, W., \& Li, F. (2019). Exosomes derived from human umbilical cord mesenchymal stromal cells deliver exogenous miR-145-5p to inhibit pancreatic ductal adenocarcinoma progression. Cancer Letters, 442, 351-361.

134. Kamerkar, S., LeBleu, V. S., Sugimoto, H., Yang, S., Ruivo, C. F., Melo, S. A., Lee, J. J., \& Kalluri, R. (2017). Exosomes facilitate therapeutic targeting of oncogenic KRAS in pancreatic cancer. Nature, 546, 498-503.

135. Xie, Y., Hang, Y., Wang, Y., Sleightholm, R., Prajapati, D. R., Bader, J., Yu, A., Tang, W., Jaramillo, L., Li, J., Singh, R. K., \& Oupicky, D. (2020). Stromal modulation and treatment of metastatic pancreatic cancer with local intraperitoneal triple miRNA/ siRNA nanotherapy. ACS Nano, 14, 255-271.

136. Shang, S., Wang, J., Chen, S., Tian, R., Zeng, H., Wang, L., Xia, M., Zhu, H., \& Zuo, C. (2019). Exosomal miRNA-1231 derived from bone marrow mesenchymal stem cells inhibits the activity of pancreatic cancer. Cancer Medicine, 8, 7728-7740.

137. Wu, D. M., Wen, X., Han, X. R., Wang, S., Wang, Y. J., Shen, M., Fan, S. H., Zhang, Z. F., Shan, Q., Li, M. Q., Hu, B., Lu, J., Chen, G. Q., \& Zheng, Y. L. (2019). Bone marrow mesenchymal stem cell-derived exosomal MicroRNA-126-3p inhibits pancreatic cancer development by targeting ADAM9. Mol Ther Nucleic Acids, $16,229-245$

138. Luan, X., Sansanaphongpricha, K., Myers, I., Chen, H., Yuan, H., \& Sun, D. (2017). Engineering exosomes as refined biological nanoplatforms for drug delivery. Acta Pharmacologica Sinica, 38, 754-763.

139. Elsharkasy, O. M., Nordin, J. Z., Hagey, D. W., de Jong, O. G., Schiffelers, R. M., Andaloussi, S. E., \& Vader, P. (2020). Extracellular vesicles as drug delivery systems: Why and how? Advanced Drug Delivery Reviews, 159, 332-343.

140. Zuo, L., Tao, H., Xu, H., Li, C., Qiao, G., Guo, M., Cao, S., Liu, M., \& Lin, X. (2020). Exosomes-coated miR-34a displays potent antitumor activity in pancreatic cancer both in vitro and in vivo. Drug Des Devel Ther, 14, 3495-3507.

141. Chu, N. J., Anders, R. A., Fertig, E. J., Cao, M., Hopkins, A. C., Keenan, B. P., Popovic, A., Armstrong, T. D., Jaffee, E. M., \& Zimmerman, J. W. (2020). Inhibition of miR-21 regulates mutant KRAS effector pathways and intercepts pancreatic ductal adenocarcinoma development. Cancer Prevention Research (Philadelphia, Pa.), 13, 569-582.

142. Gilles, M. E., Hao, L., Huang, L., Rupaimoole, R., Lopez-Casas, P. P., Pulver, E., Jeong, J. C., Muthuswamy, S. K., Hidalgo, M., Bhatia, S. N., \& Slack, F. J. (2018). Personalized RNA Medicine for Pancreatic Cancer. Clinical Cancer Research, 24, 1734-1747.

143. Fei, X., Jin, H. Y., Gao, Y., Kong, L. M., \& Tan, X. D. (2020). Hsa-miR-10a-5p promotes pancreatic cancer growth by BDNF/ 
SEMA4C pathway. Journal of Biological Regulators and Homeostatic Agents, 34, 927-934.

144. Kong, F., Li, L., Wang, G., Deng, X., Li, Z., \& Kong, X. (2019). VDR signaling inhibits cancer-associated-fibroblasts' release of exosomal miR-10a-5p and limits their supportive effects on pancreatic cancer cells. Gut, 68, 950-951.

145. Xiong, G., Huang, H., Feng, M., Yang, G., Zheng, S., You, L., Zheng, L., Hu, Y., Zhang, T., \& Zhao, Y. (2018). MiR-10a-5p targets TFAP2C to promote gemcitabine resistance in pancreatic ductal adenocarcinoma. Journal of Experimental \& Clinical Cancer Research, 37, 76.

146. Sheedy, P., \& Medarova, Z. (2018). The fundamental role of miR$10 \mathrm{~b}$ in metastatic cancer. American Journal of Cancer Research, $8,1674-1688$

147. Xu, C., \& Qi, X. (2020). MiR-10b inhibits migration and invasion of pancreatic ductal adenocarcinoma via regulating E2F7. J Clin Lab Anal, 34, e23442.
148. Yoo, B., Greninger, P., Stein, G. T., Egan, R. K., McClanaghan, J., Moore, A., Benes, C. H., \& Medarova, Z. (2018). Potent and selective effect of the mir-10b inhibitor MN-anti-mir10b in human cancer cells of diverse primary disease origin. PLoS One, 13, e0201046.

149. Yoo, B., Jordan, V. C., Sheedy, P., Billig, A. M., Ross, A., Pantazopoulos, P., \& Medarova, Z. (2019). RNAi-mediated PD-L1 inhibition for pancreatic cancer immunotherapy. Science and Reports, 9 , 4712 .

Publisher's note Springer Nature remains neutral with regard to jurisdictional claims in published maps and institutional affiliations. 